\title{
Estudio de la resistencia a la tracción de depósitos de soldadura de la aleación AA2024-T3*
}

| Fecha de recibido: 20 de marzo del 2021 | Fecha de aprobación: 13 de abril del 2021 |

\section{David Alberto Ramírez Vargas \\ Estudiante de maestría}

Universidad Nacional de Colombia Colombia

Grupo de Investigación: Análisis de Falla

e Integridad de Superficies (AFIS)

Rol de investigador: teórico y escritura

https://orcid.org/0000-0003-3045-8752

$\bowtie$ daaramirezva@unal.edu.co

\section{Andrés Fernando Gil Plazas}

Ingeniero mecánico

Universidad Nacional de Colombia Colombia

Grupo de Investigación: Análisis de Falla e Integridad de Superficies (AFIS) y Grupo de investigación del Centro de

Materiales y Ensayos (GIMES, SENA) Rol de investigador: teórico y experimental https://orcid.org/0000-0001-6585-9121

$\bowtie$ agilp@unal.edu.co
Liz Karen Herrera Quintero

Doctora en Ciencia de Materiales

Universidad Nacional de Colombia Colombia Grupo de Investigación: Análisis de Falla e Integridad de Superficies (AFIS)

Rol de investigador: teórico https://orcid.org/0000-0003-2002-4336 $\bowtie$ Ikherreraq@unal.edu.co

* Artículo de investigación resultado de la tesis de maestría vinculada al grupo de investigación de Análisis de fallas e integridad estructural, Facultad de Ingeniería de la Universidad Nacional de Colombia, Sede Bogotá.

Cómo citar este artículo: Ramírez Vargas, D. A., Gil Plazas, A. F., \& Herrera Quintero, L. K. (2021). Estudio de la resistencia a la tracción de depósitos de soldadura de la aleación AA2024-T3. Ciencia y Poder Aéreo, 16(1). 18- 38, https://doi.org/10.18667/cienciaypoderaereo.708 


\section{Estudio de la resistencia a la tracción de depósitos de soldadura de la aleación AA2024-T3}

\section{Study of Weld Deposits Tensile Strength in the \\ AA2024-T3 Alloy}

Resumen: En el presente artículo, se realiza un estudio de la resistencia a la tracción de diferentes depósitos de soldadura sobre láminas de la aleación de aluminio AA2024-T3 AlClad. La soldabilidad de la aleación, bajo procesos de arco, se considera limitada por su sensibilidad al agrietamiento en caliente y, por ello, se encuentra ampliamente estudiada en procesos de estado sólido o láser, en los que la afectación térmica es menor. La investigación tiene como objetivo estudiar el comportamiento mecánico de dicho aluminio al soldarlo con tecnología local. Para la experimentación, se realizaron depósitos con combinaciones de electrodo no consumible punto violeta, electrodo no consumible punto verde, metal de aporte ER4043 y sin metal de aporte (soldadura autógena). Los ensayos mecánicos estuvieron bajo el estándar ASTM E8 y bajo las recomendaciones del estándar AWS D1.2. Adicionalmente, se realizó el respectivo estudio de las superficies de fractura de cada uno de los depósitos. Entre los resultados, se destaca la notable reducción tanto de resistencia mecánica como de ductilidad, lo cual se debió a la alta presencia de porosidad, que desencadenó grietas de diversos tamaños, ligados, también, a una fragilización por hidrógeno y por presencia de partículas de segunda fase en los límites de grano.

Palabras clave: resistencia mecánica; GTAW-P; fractura; agrietamiento en caliente

Abstract: This paper examines the tensile strength of different weld deposits on sheets of the AA2024-T3 AlClad aluminum alloy. The weldability of this alloy under arc welding processes is considered limited due to its sensitivity to hot cracking. Therefore, it has been widely studied in solid-state or laser processes in which the thermal impact is reduced. The research aims to study the mechanical behavior of said aluminum alloy when local technology is used for welding. Experimentation included the use of deposits elaborated with combinations of non-consumable violet point electrode, non-consumable green point electrode, ER4043 filler metal, and without filler metal (autogenous welding). Mechanical tests were performed under the ASTM E8 standard and following the recommendations of the AWS D1.2 standard. The corresponding study of fractured surfaces in each deposit was also carried out. Results show a considerable reduction in both mechanical resistance and ductility as a result of the strong presence of porosity, which triggered cracks of various sizes, also linked to embrittlement by hydrogen and the presence of second-phase particles at grain boundaries.

Keywords: Mechanical strength; GTAW-P; fracture; hot cracking.

Resumo: No presente artigo, é realizado um estudo da resistência à tração de diferentes reservatórios de soldagem sobre lâminas de liga de alumínio AA2024-T3 Alclad. A soldabilidade da liga, sob processos de arco, é considerada limitada pela sua sensibilidade à fissuração no quente, e, por isso, se encontra amplamente estudada em processos de estado sólido ou a laser, em que a afetação térmica é menor. A investigação tem por objetivo estudar o comportamento mecânico do referido alumínio ao soldá-lo com tecnologia local. Para a experimentação, foram realizados depósitos com combinações de eletrodo não consumível ponto violeta, eletrodo não consumível ponto verde, metal de entrada ER4043 e sem metal de entrada (soldagem autônoma). Os ensaios mecânicos estiveram sob o padrão ASTM E8 e sob as recomendações da norma AWS D1.2. Adicionalmente, realizou-se o respectivo estudo das superfícies de fratura de cada um dos depósitos. Entre os resultados, destaca-se a notável redução tanto de resistência mecânica como de ductilidade, o que se deveu à alta presença de porosidade, que desencadeou fissuras de diversos tamanhos, ligados, também, a uma fragilização por hidrogénio e à presença de partículas de segunda fase nos limites do grão.

Palavras-chave: resistência mecânica, GTAW-P, fratura, fissuração em quente 
Hoy en día, el aluminio representa cerca del $80 \%$ del peso de las construcciones de aeronaves comerciales y el $50 \%$ de aeronaves militares, en las que también coexisten las aleaciones de magnesio y las superaleaciones. Inicialmente, se trabajó con aleaciones 2017; después, con la 2024 y, en la actualidad, las aleaciones de las series 6 XXX y 7XXX son las más usadas. La aleación 2024 fue introducida en la década de 1930 como una aleación de envejecimiento natural con mayor resistencia mecánica; fue desarrollada para reemplazar la aleación 2017 (Liu et al., 2019; Mouritz, 2012).

Con el fin de mejorar los tiempos de mantenimiento y reducir gastos en el reemplazo de partes, se han estudiado diversas formas en que estos duraluminios pueden ser soldados. En general, las investigaciones apuntan a que la forma más efectiva de soldar los aluminios de la serie $2 X X X$ es a través de procesos de soldadura en estado sólido, específicamente la soldadura por fricción (FSW) y la soldadura láser. Además, se centran en el estudio del efecto que tiene la entrada de calor sobre las propiedades mecánicas (Hashimoto et al., 2016; Liang et al., 2020; Soysal \& Kou, 2019).

Fu et al. (2013) investigaron la dureza en la zona de agitación (sz, por sus siglas en inglés), para diferentes entradas de calor, de juntas sin tratamiento térmico posterior a la soldadura, denominado as-welded, y con envejecido natural posterior al procedimiento de soldadura (PWNA). Entre sus hallazgos, destacan que, con una alta entrada de calor, la dureza de la sz es menor que la del metal base; sin embargo, una baja entrada de calor no influye de forma directa en la dureza, sino que se presenta una alta dependencia de la velocidad de rotación: con un incremento en la velocidad de rotación, aumenta la dureza de dicha zona. También encontraron que no es necesario un PWNA, pues, con un adecuado control del pico de temperatura y la respectiva tasa de enfriamiento, es suficiente para alcanzar valores cercanos al metal base.

De forma análoga, Chen et al. (2015) investigaron el efecto de la entrada de calor sobre la dureza de la sz, pero con un tratamiento térmico posterior a la soldadura (PWAA). Los hallazgos son similares a los de Fu et al. (2013), al identificar que una alta entrada de calor, junto con una elevada velocidad de avance, representa mejoras en la dureza de la sz, y que, a baja entrada de calor, es necesario incrementar la velocidad de rotación. En este caso, el tratamiento térmico posterior a la soldadura implicó una reducción en la dureza de la junta, a causa del sobreenvejecimiento que se presentó.

Jones et al. (2005) reportaron dos zonas de dureza mínima dentro de la zona afectada por el calor. La más cercana a la zona afectada termomecánicamente (TMAZ) se debió al sobreenvejecido de la fase S, en el ciclo térmico, mientras que la zona de mínima dureza exterior fue a causa de la disolución de la fase $\mathrm{S}$, responsable en mayor parte del endurecimiento del metal base (Wang et al., 2017).

Ahora, en cuanto a la unión de aleaciones disímiles, de las cuales la más común es la de la aleación AA2024 con la AA7075, Cavaliere et al. (2006) estudiaron el comportamiento mecánico y microestructural de la unión de láminas de estos dos materiales y observaron cómo la presencia de dendritas equiaxiales se fue reduciendo desde las zonas de recristalización hacia el centro de la junta. Sobre la resistencia mecánica, encontraron lo típico de una unión de materiales disímiles, esto es, ruptura en la zona afectada por el calor del material con menor resistencia (AA2024) y reducción en la vida a fatiga con disminución en la amplitud y elevada pendiente en la curva S-N. Por su parte, Gowthaman y Saravanan (2020), usando también aleaciones AA2024 y AA7075, experimentaron con combinaciones de la velocidad de rotación y velocidad de avance, con fuerza axial constante, y reportaron una combinación favorable cuando se tiene baja velocidad de avance y alta velocidad de rotación, lo cual produce una alta resistencia a la tracción.

Sobre la soldadura de la aleación por arco, como Gas Metal Arc Welding (GMAW, también conocida como MIG) y Gas Tungsten Arc Welding (GTAW O TIG), la información es bastante limitada, y se estudia principalmente la serie 6XXX y la soldadura de aleaciones disímiles. Norman et al. (1999) ejecutaron soldadura autógena con el proceso TIG para diferentes combinaciones de parámetros de soldadura. Determinaron que una combinación de altas velocidades de avance y bajas densidades de corriente otorgan las condiciones térmicas necesarias para la nucleación y crecimiento 
de granos equiaxiales en el charco de soldadura. Además, observaron cómo en el centro de la soldadura se presenta una microestructura refinada, atribuida a las altas tasas de enfriamiento que operan en esa región.

Las soldaduras de materiales disímiles por arco son preferentes en las aleaciones AA6063 y AA7075. En concreto, Maamar et al. (2008) e Hima Bindu et al. (2020) realizaron estudios sobre la soldabilidad con la aleación AA7075. Maamar et al. encontraron que los granos del material base, adyacentes a la línea de fusión, presentan embastecimiento de los granos cristalizados y una migración de las partículas intermedias de segunda fase hacia sus límites, caso atribuible a la baja disipación de calor generado durante el proceso. Hima Bindu et al., usando aporte ER5356, fueron testigos del ya mencionado fenómeno de embastecimiento del grano con el incremento de la corriente, ocasionando un aumento en la dureza y, al igual que Maamar et al., encontraron segregación de partículas de segunda fase hacia los límites de grano, que resultaron en grietas intergranulares.

Por otro lado, Vijay et al. (2020), con su procedimiento de soldadura de las aleaciones AA2024 y AA6063, encontraron un posible incremento en la resistencia a la tracción y en la dureza por el incremento en la entrada de calor, el flujo de gas y la apertura de raíz. Por ello, es importante considerar la temperatura a la que puede llegar el material y el arco a causa del procedimiento de soldadura, ya que eso determina el comportamiento final de la soldadura y no será medida en el experimento. Por ejemplo, Esfahani et al. (2018), con su estudio sobre soldadura de aluminio puro bajo GTAW, indican que la zona afectada por el calor experimenta temperaturas entre $377 \mathrm{~K}\left(100^{\circ} \mathrm{C}\right)$ y $643 \mathrm{~K}\left(370^{\circ} \mathrm{C}\right)$. Du et al. (2019), en sus simulaciones y comparaciones de los gradientes de temperatura de la soldadura por arco sobre la aleación AA2024 de espesor $6 \mathrm{~mm}$, indicaron que la temperatura del depósito en el punto de contacto con el electrodo es de casi $990 \mathrm{~K}\left(718^{\circ} \mathrm{C}\right)$ y, a $2 \mathrm{~mm}$, la isoterma estará cerca de los $600 \mathrm{~K}\left(327^{\circ} \mathrm{C}\right)$.

En algunos estudios se comparan estos dos procesos de soldadura. Por ejemplo, Mohapatra y Sarangi (2016) y Squillace et al. (2004) compararon las técnicas
FSW y GTAW en el aspecto microestructural y de resistencia a la corrosión por pitting. Ambos concuerdan en que, para el proceso GTAW, la dureza cae tanto en la zona afectada por el calor como en el metal de soldadura, en relación con la alta temperatura que se induce por el proceso en sí; mientras que, para Fsw, la sz y la TMAZ presentan una caída en las propiedades mecánicas de resistencia a la tracción y dureza. Además, se pudo establecer que, para la HAZ, hay una ligera recuperación de estas propiedades mecánicas.

Cabe resaltar que hay técnicas nuevas y novedosas en la unión de estos metales, que implican una baja entrada de calor y evitan algunos de los problemas expuestos. Ahn et al. (2017) realizaron un estudio sobre los efectos de usar argón y helio en la soldadura por fibra láser, analizando la calidad en términos de ancho de cara, ancho de raíz socavado, falta de llenado y porosidad. Su principal conclusión es que, si bien con el helio y su alto poder de ionización se consigue un ancho adecuado a bajas velocidades, el argón ofreció una mejor calidad en el depósito. También analizaron el efecto de la tasa de alimentación del aporte sobre la composición y microestructura de la aleación soldada por fibra láser, encontrando que, en un intervalo de 2 a $3 \mathrm{~m} / \mathrm{min}$, se consiguió un radio de dilución de $9 \%$ en un depósito con $0,6 \%$ de silicio, reduciendo el compuesto Mg2Si y, por ende, minimizando el riesgo de agrietamiento. Por su parte, Prakash et al. (2018) demostraron mejoras significativas de dureza y resistencia a la tracción, haciendo uso de la técnica Cold Metal Transfer (CMT).

En términos generales, la soldabilidad es relativamente reducida para un grupo amplio de aleaciones de aluminio, en el que las soldaduras tienden a ser frágiles y la fusión eutéctica alrededor de estas debilita la junta. Los refinadores de grano, como el titanio, el boro y el circonio, reducen la tendencia al agrietamiento (Mondolfo, 1976).

Ahora bien, características propias del aluminio, como su capa de óxido, su elevada conductividad térmica, su considerable coeficiente de dilatación térmica, los altos rangos de solidificación que experimenta y la alta solubilidad del hidrógeno en estado líquido, hacen de la soldadura del aluminio una tarea desafiante. 
Cubrir todos los problemas potenciales al tiempo es complicado, pero acciones como controlar la entrada de calor y la velocidad de avance y los tratamientos previo y posterior a la soldadura han demostrado la reducción de sus efectos negativos (Qi et al., 2019).

La combinación del alto coeficiente de expansión térmica, la contracción de solidificación y un amplio intervalo de temperaturas de solidificación desembocan en una sensibilidad al agrietamiento (ASM International, 2020b; Hatch, 1984). En este caso, un grano refinado hace que la película del líquido intergranular residual se reduzca, provocando una mayor cohesión de los granos, mitigando así el agrietamiento por solidificación, lo que mejora las propiedades mecánicas. En general, granos grandes se producen a causa de la baja disipación del calor generado. En ese sentido, el tamaño del grano aumentará con el aumento en la temperatura pico. El máximo pico de temperatura en la HAZ es en la frontera de la zona de fusión, por lo que esta es la responsable de la variación del tamaño del grano posterior al proceso de soldadura (Esfahani et al., 2018; Kou \& Le, 1988; Lancaster, 1999; Maamar et al., 2008; Vijay et al., 2020).

La porosidad es difícil de controlar, debido a la alta difusividad del hidrógeno en el aluminio líquido, la interacción con el ambiente y la alta tasa de solidificación. La difusión del hidrógeno puede llegar a ser tan alta como $1 \mathrm{~cm}^{3} / \mathrm{g}$, dando paso a la formación de burbujas. Según Ambriz y Jaramillo (2014), las burbujas deben tener un radio crítico mayor a $700 \mu \mathrm{m}$ para que puedan sobreponerse a la tensión superficial y alcanzar la superficie con ayuda de la fuerza de empuje. Sin embargo, en un proceso real, el ciclo térmico de enfriamiento de la zona de fusión es muy rápido, por lo que es complicado, en algunos casos, llegar a ese estado de equilibrio. En realidad, se requiere de una tasa de solidificación muy baja para que la burbuja se forme y alcance su valor crítico. Al mismo tiempo, el grado de degradación de la zona afectada por el calor puede variar considerablemente si se lleva un control sobre las variables del proceso, tomando en consideración la influencia del ancho de esta zona del espesor del material, la velocidad de avance y la entrada de calor (ASM International, 2020b; Mathers, 2002).
De acuerdo con lo anterior, la investigación se centra en determinar qué tan factible es soldar un aluminio 2024-T3 con la tecnología disponible en Colombia. Para ello, se ejecutaron diferentes combinaciones de variables como electrodo no consumible y método de aporte, con el fin de evaluar la resistencia a la tracción de los depósitos. El estudio se complementa con el estudio de la superficie de fractura para, por un lado, determinar los diferentes modos y mecanismos de falla que se pueden presentar a causa de la combinación de variables y, por otro, contrastarlos con los tipos de fractura propuestos por Matsuda (Lippold, 2015).

\section{Metodología}

Para la experimentación, se llevaron a cabo diferentes depósitos de soldadura en láminas de la aleación de aluminio AA2024 en su estado de entrega T3 con AlClad, de acuerdo con la composición química indicada en la tabla 1. Las propiedades mecánicas de referencia se encuentran resumidas en la tabla 2.

Tabla 1

Composición química teórica de la aleación AA2024-T3

\begin{tabular}{c|c|c|c|c|c|c}
\hline Al & $\mathbf{S i}$ & $\mathbf{F e}$ & $\mathbf{C u}$ & $\mathbf{M n}$ & $\mathbf{M g}$ & $\mathbf{G r}$ \\
\hline Balance & 0.5 & 0.5 & $3.8-4.9$ & $0.3-0.9$ & $1.2-1.8$ & 0.1 \\
\hline
\end{tabular}

Fuente: elaboración propia, a partir de ASM International (2020a)

Tabla 2

Propiedades mecánicas reportadas de la aleación AA2024-T3

\begin{tabular}{l|c|c|c|c|c|c}
$\begin{array}{l}\text { Aleación y } \\
\text { tratamiento }\end{array}$ & \multicolumn{2}{|c|}{$\begin{array}{c}\text { Resistencia } \\
\text { última }\end{array}$} & \multicolumn{2}{|c|}{$\begin{array}{c}\text { Resistencia } \\
\text { a la fluencia }\end{array}$} & $\begin{array}{c}\text { Elongación } \\
\text { en 50 mm, \% }\end{array}$ & $\begin{array}{c}\text { Dureza } \\
\text { HB }\end{array}$ \\
\cline { 2 - 6 } & $\mathbf{M P a}$ & $\mathbf{k s i}$ & $\mathbf{M P a}$ & $\mathbf{k s i}$ & & 120 \\
\hline $\begin{array}{l}\text { AlClad } \\
\text { 2024-T3 }\end{array}$ & 450 & 65 & 310 & 45 & 18 & 120 \\
\hline
\end{tabular}

Fuente: elaboración propia, a partir de ASTM B209-14 (2014)

Para los depósitos, se escogió un proceso GTAW con alta frecuencia y se trabajó con corriente alterna pulsada. Variables como velocidad de avance, corriente y voltaje se dejaron constantes para todos los 
depósitos. Se trabajó con una trimezcla de gases de 200 ppm de óxido nitroso, 200 ppm de oxígeno y balance argón, que se asemeja a una mezcla de $20 \%$ de helio, según lo indica su patente (Miller et al., 2013). Las variables específicas del procedimiento pueden ser consultadas en la tabla 3. El recubrimiento AlClad fue retirado mediante cepillado mecánico, con el fin de mitigar las posibles inclusiones que puedan desencadenar en los depósitos (Tier et al., 2009).

Tabla 3

Variables del proceso de soldadura por GTAW-HF

\begin{tabular}{c|c|c|c|c|c|c|c|}
$\begin{array}{c}\text { Voltaje } \\
\text { (V) }\end{array}$ & $\begin{array}{c}\text { Corriente } \\
\text { pico (I) }\end{array}$ & \multicolumn{2}{|c|}{$\begin{array}{c}\text { Velocidad } \\
\text { de avance (v) }\end{array}$} & $\begin{array}{c}\text { Entrada de } \\
\text { calor (0) }\end{array}$ & \multicolumn{2}{|c|}{$\begin{array}{c}\text { Tasa de entrada } \\
\text { de calor (q) }\end{array}$} & $\begin{array}{c}\text { Flujo } \\
\text { de gas }\end{array}$ \\
\hline$[\mathrm{V}]$ & {$[\mathrm{A}]$} & {$[\mathrm{in} / \mathrm{min}]$} & {$[\mathrm{mm} / \mathrm{s}]$} & {$[\mathrm{W}]=[\mathrm{J} / \mathrm{s}]$} & {$[\mathrm{kJ} / \mathrm{in}]$} & {$[\mathrm{J} / \mathrm{mm}]$} & {$[\mathrm{L} / \mathrm{min}]$} \\
\hline 10.0 & 55.0 & 7.0 & 3.0 & 385.0 & 3.3 & 129.9 & 10.0 \\
\hline
\end{tabular}

Fuente: elaboración propia.

La junta se trató de un bisel recto a tope sin separación de raíz, tal como se indica en la figura 1 , de acuerdo con los estándares Aws A2.4 y Aws A3.0 (American Welding Society, 2020a, 2020b). Se trabajaron cuatro combinaciones de variables a partir de la variación del metal de aporte (con aporte ER4043 y sin aporte) y del electrodo no consumible (electrodo punto verde y electrodo punto violeta). Con el fin de obtener un adecuado número de probetas para analizar, se realizaron dos repeticiones de cada combinación, para un total de ocho cupones - tabla 4-. Cabe resaltar que se extrajeron dos probetas de tensión por depósito, de acuerdo con lo exigido por el código de calificación Aws D1.2 (American Welding Society, 2014).

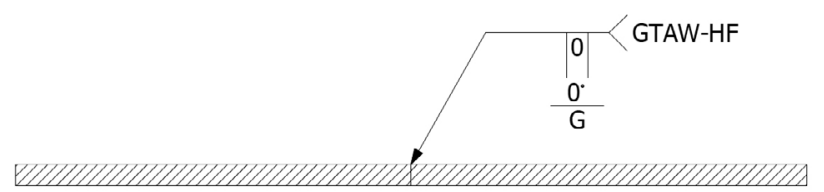

Figura 1. Esquema de junta a tope diseñada para el estudio Fuente: elaboración propia.
Tabla 4

Depósitos de soldadura, agrupados por el material de aporte y electrodo no consumible

\begin{tabular}{c|l|l|l}
$\begin{array}{c}\mathbf{N}^{\mathbf{0}} \\
\text { depósito }\end{array}$ & $\begin{array}{c}\text { Material de } \\
\text { aporte }\end{array}$ & $\begin{array}{c}\text { Electrodo no } \\
\text { consumible }\end{array}$ & \multicolumn{1}{|c}{ Abreviación } \\
\hline 1 & Sin Aporte & Punto verde & SAVE \\
\hline 8 & ER-4043 & $\begin{array}{l}\text { EWG - Punto } \\
\text { Violeta }\end{array}$ & CAVI \\
\hline 2 & Sin Aporte & $\begin{array}{l}\text { EWG - Punto } \\
\text { Violeta }\end{array}$ & SAVI \\
\hline 7 & ER-4043 & Punto verde & CAVE \\
\hline 5 & &
\end{tabular}

Nota. El número de depósito corresponde al orden en el que se ejecutó la soldadura, después de la aleatorización.

Fuente: elaboración propia.

Las respectivas probetas fueron maquinadas, tal como se indica en la figura 2, con una longitud calibrada de 50,8 mm (2 in) y sometidas a carga uniaxial en una máquina universal de ensayos SHIMADZU UH50A de $50 \mathrm{kN}$, con una velocidad de avance del cabezal de $5 \mathrm{~mm} / \mathrm{min}$. El procedimiento de ensayo siguió las especificaciones del estándar ASTM E8 (ASTM E8/E8M-21, 2021) y del estándar Aws B4,0 (American Welding Society, 2016).

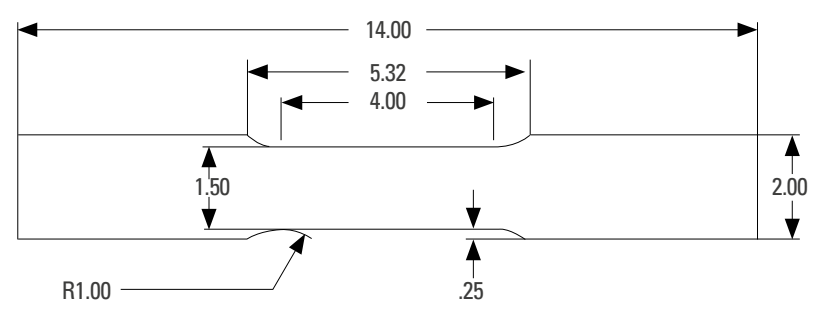

Figura 2. Esquema de las probetas usadas para el ensayo de tensión. Unidades en pulgadas

Fuente: elaboración propia.

Para la observación y el análisis de las respectivas superficies de fractura, se utilizó un estereoscopio Discovery V.12 y un microscopio electrónico de barrido Phenom xL, al que se le acopló una sonda para análisis semicuantitativo de composición química de puntos sobre la superficie. 
Para la observación y el análisis de las respectivas superficies de fractura, se utilizó un estereoscopio Discovery V.12 para identificar cualitativamente el modo de falla. Por microscopia electrónica de barrido (SEM) con el equipo Phenom XL, con acople de una sonda para microanálisis semicuantitativo de composición química (EDS), se observó la superficie de fractura con el ánimo de observar con mayor profundidad de campo y resolución la rugosidad presente en las probetas. También, mediante la emisión característica de rayos $X$ de cada elemento, se reconoce la presencia de los elementos, asociándolos a la segregación o presencia de las partículas de segunda fase presentes y cómo influyen en su respuesta mecánica.

\section{Resultados}

\section{Depósitos con electrodo punto verde y sin metal de aporte (SAVE)}

El resultado de los ensayos de tracción para las probetas 1 y 8 , correspondientes a la combinación de electrodo no consumible punto verde sin metal de aporte, se encuentra en las curvas de esfuerzo-deformación de la figura 3 y en la tabla 5.

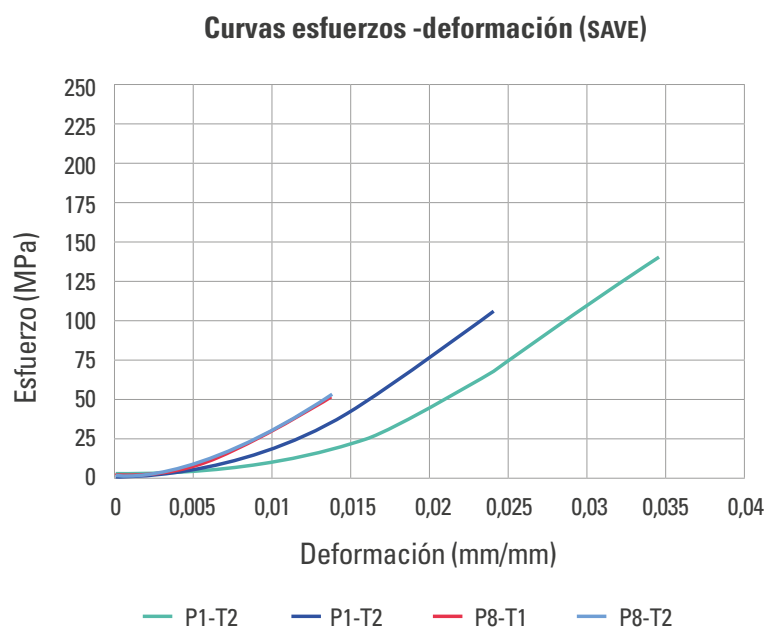

Figura 3. Curvas esfuerzo-deformación combinación sin metal de aporte y electrodo punto verde

Fuente: elaboración propia.
Las probetas del depósito 8 mostraron un comportamiento similar entre sí. Por el contrario, las probetas del depósito 1 variaron entre sí en más del $32 \%$ en resistencia y $43 \%$ en ductilidad, lo que se considera como una inconsistencia debido a una posible variación en condiciones como la velocidad de avance o entrada de calor de la soldadura (por cambio en la distancia de arco), situación típica en procesos de soldadura manuales. Es evidente, también que, en ninguno de los cuatro ensayos, hubo una transición entre comportamiento elástico y plástico, asemejándose las curvas a las de un material frágil.

En términos generales, se evidencia una notable reducción, tanto en la resistencia última a la tracción como en la ductilidad, en los cuatro casos con respecto a los valores de referencia de 450 MPa de UTS y $18 \%$ de alargamiento.

Tabla 5

Resumen esfuerzo último y alargamiento de la combinación sin metal de aporte y electrodo punto verde

\begin{tabular}{c|c|c|c}
\hline \multicolumn{4}{|c}{ SAVE } \\
\hline Depósito & Muestra & UTS [MPa] & Alargamiento \\
\hline 1 & T1 & 138,88 & $3,45 \%$ \\
\hline 1 & T2 & 104,49 & $2,40 \%$ \\
\hline 8 & T1 & 50,85 & $1,37 \%$ \\
\hline 8 & T2 & 52,08 & $1,37 \%$ \\
\hline
\end{tabular}

Fuente: elaboración propia.

Al realizar la respectiva fractografía, en las macrografías de las figuras 4(a) y 4(b) se ve la alta concentración de porosidad en los depósitos de soldadura, donde, por la coloración amarilla, se percibe una corrosión. La superficie de fractura tiene aspecto granular, con una reflectividad y rugosidad apreciable, sin marcas aparentes de origen ni direcciones de propagación de la grieta, relacionándose con una fractura súbita frágil (ASM International, 2002). No hay rastros aparentes de deformación plástica.

Con la observación de la superficie a 500 aumentos -figura 4(c) - , se presume una fractura súbita mixta por la aparente existencia de microvacíos, junto con una trayectoria intergranular, lo que, de acuerdo con 
ASM International (2002), se relaciona con una presencia parcial de elementos fragilizantes en los límites de grano, debido a la segregación. Con la micrografía a 1.000 aumentos, se aprecian ciertos hoyuelos en los brazos de las dendritas que se formaron. En esta imagen, se puede apreciar cómo el tipo de fractura se asemeja al Tipo D-F, por tratarse de una transición entre el dendrítico y plano (Lippold, 2015). En ambas imágenes es evidente la formación de porosidades de solidificación.

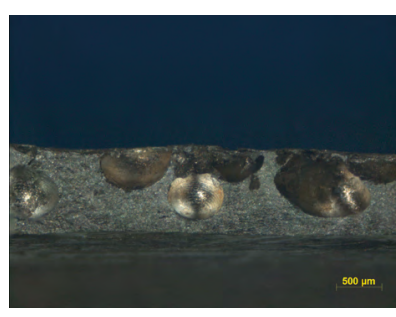

(a)

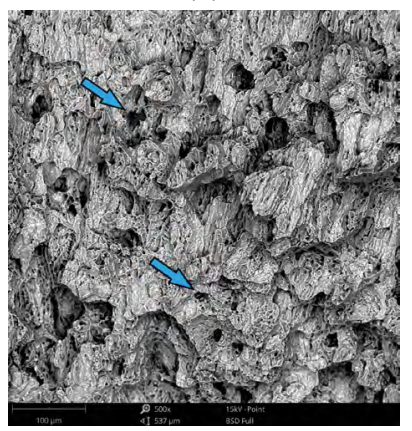

(c)

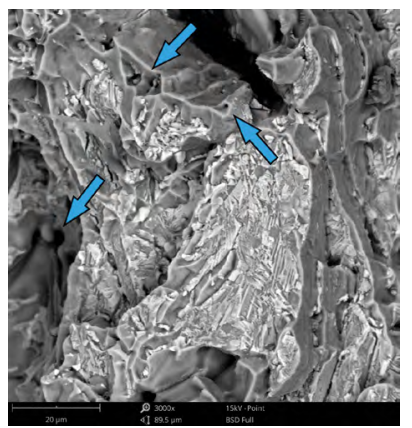

(e)

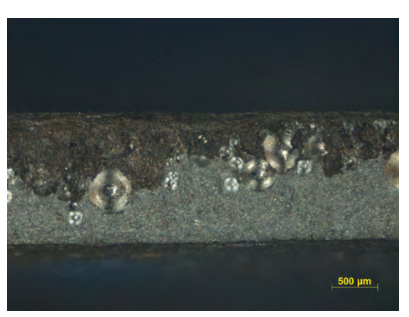

(b)

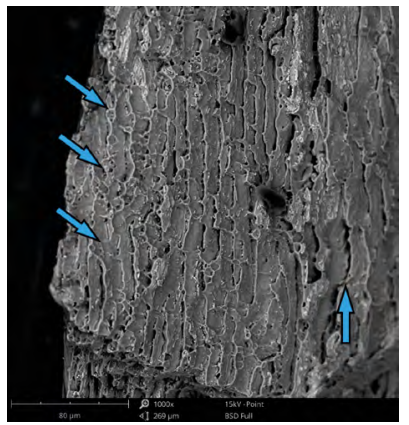

(d)

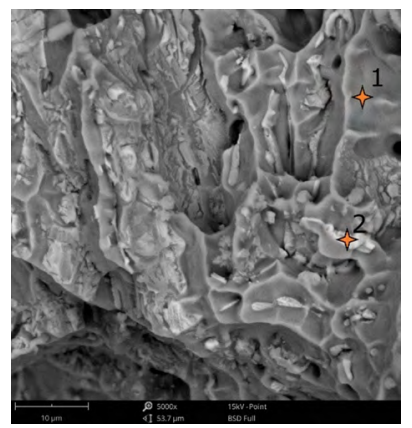

(f)
Figura 4. Superficies de fractura de la combinación sin metal de aporte y electrodo punto verde

Nota. Descripción de las imágenes: (a) macrografía de la superficie de fractura de la probeta 1 a 200X; (b) macrografía de la superficie de fractura de la probeta 8 a 200X; (c) micrografía de la superficie de fractura de la probeta 8 a 500X;

(d) micrografía de la superficie de fractura de la probeta 1 a 1000X; (e) micrografía de la superficie de fractura de la probeta 8 a 3000X; (f) micrografía de la superficie de fractura de la probeta 8 a 5000X, con la indicación de los puntos para análisis mediante SEM/EDS.

Fuente: elaboración propia.
Con mayores aumentos, como los presentados en las figuras $4(\mathrm{e})$ y $4(\mathrm{f})$, se evidencia nuevamente la porosidad de contracción; pero, esta vez, se observa una trayectoria intergranular, en la que se ve un modo de fractura por descohesión (ASM International, 2002), posiblemente, a causa de los elementos que se alojan en algunos hoyuelos producto de la separación. Cabe resaltar la presencia de una capa, de lo que puede considerarse líquido eutéctico, que cubre parcialmente la superficie de fractura.

Mediante el análisis de composición elemental de los puntos indicados en la figura 4(f), se determina, a partir de la composición del punto 1 , presentada en la tabla 6, que, por su alto contenido de aluminio, se trata de un elemento microestructural en solución sólida de la matriz del material. El espectro de composición elemental de este punto se presenta en la figura 5, en la que se evidencian los elementos identificados y analizados.

Ahora bien, analizando uno de los elementos que se advirtieron en los puntos de descohesión de los granos, como lo es el punto 2 de la figura 4, por el contenido de aluminio y cobre presentado en la tabla 7 , se infiere que es un compuesto intermetálico del tipo $\mathrm{Al}-\mathrm{Cu}-\mathrm{Mg}$, que, para las relaciones $\mathrm{Cu} / \mathrm{Mg}$ de $2.81 \mathrm{y} \mathrm{Mg/}$ Si de 3.2 se consideran normales (Mondolfo, 1976) y que, a su vez, actuaron en ese punto como nucleadores de grieta. El respectivo espectro de composición elemental del punto 2 se presenta en la figura 6 .

Tabla 6

Composición química elemental del punto 1 de la superficie de fractura de la combinación sin metal de aporte y electrodo punto verde

\begin{tabular}{l|c|c|c}
\hline \multicolumn{2}{c|}{ Elemento Ouímico } & \multicolumn{2}{c}{ Concentración } \\
\hline Nombre & Símbolo & Atómica \% & Peso \% \\
\hline Aluminio & $\mathrm{Al}$ & 95,97 & 93,89 \\
\hline Magnesio & $\mathrm{Mg}$ & 2,68 & 2,36 \\
\hline Cobre & $\mathrm{Cu}$ & 0,95 & 2,18 \\
\hline Plata & $\mathrm{Ag}$ & 0,40 & 1,57 \\
\hline
\end{tabular}

Fuente: elaboración propia. 


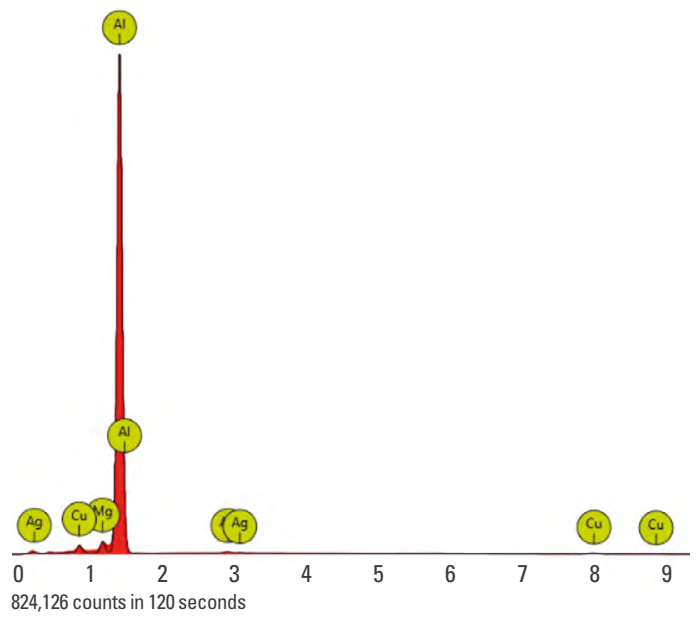

Figura 5. Espectro de composición por SEM/EDS del punto 1 de la superficie de fractura de la combinación sin metal de aporte y electrodo punto verde Fuente: elaboración propia.

Tabla 7

Composición química elemental del punto 2 de la superficie de fractura de la combinación sin metal de aporte y electrodo punto verde

\begin{tabular}{|l|c|c|c|}
\hline \multicolumn{2}{|c|}{ Elemento Químico } & \multicolumn{2}{c|}{ Concentración } \\
\hline Nombre & Símbolo & Atómica \% & Peso \% \\
\hline Aluminio & $\mathrm{Al}$ & 78,43 & 66,82 \\
\hline Cobre & $\mathrm{Cu}$ & 12,15 & 24,38 \\
\hline Magnesio & $\mathrm{Mg}$ & 7,61 & 5,84 \\
\hline Hierro & $\mathrm{Fe}$ & 0,97 & 1,71 \\
\hline Manganeso & $\mathrm{Mn}$ & 0,58 & 1,01 \\
\hline Silicio & $\mathrm{Si}$ & 0,27 & 0,24 \\
\hline
\end{tabular}

Fuente: elaboración propia.

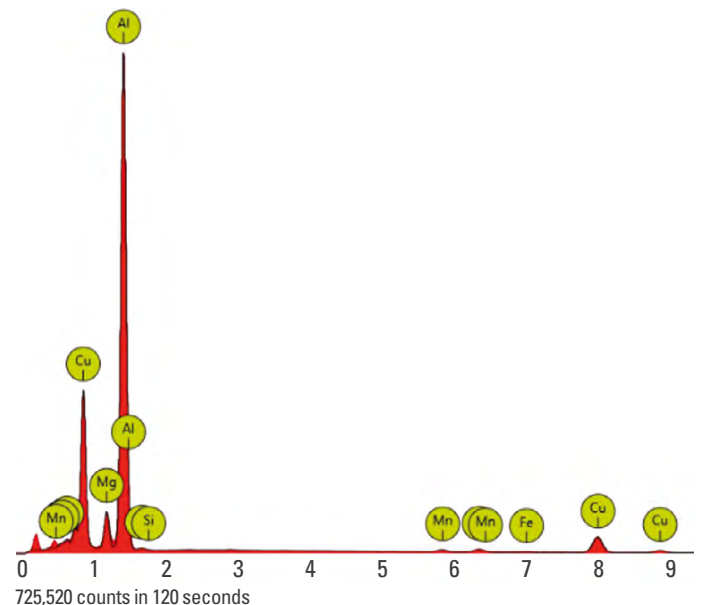

Figura 6. Espectro de composición por SEM/EDS del punto 2 de la superficie de fractura de la combinación sin metal de aporte y electrodo punto verde Fuente: elaboración propia

\section{Depósitos con electrodo punto violeta y con metal de aporte (CAVI)}

El resultado de los ensayos de tracción para las probetas 2 y 7 , correspondientes a la combinación de electrodo no consumible punto violeta con metal de aporte, se encuentra en las curvas de esfuerzo-deformación de la figura 7 y en la tabla 8.

Curvas esfuerzos -deformación (CAVI)

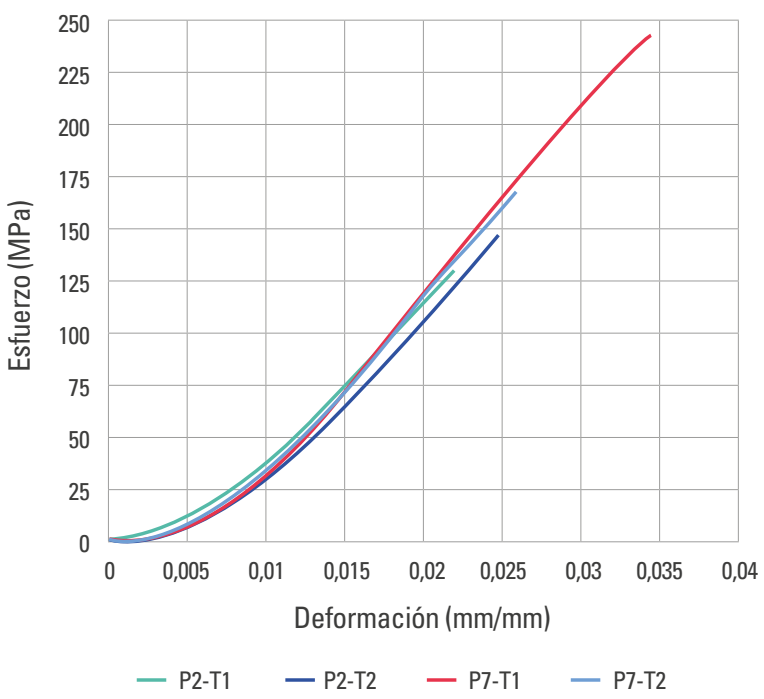

Figura 7. Curvas esfuerzo-deformación combinación con metal de aporte y electrodo punto violeta

Fuente: elaboración propia.

En esta combinación, se comprobaron valores de resistencia y ductilidad más consistentes, comparados con la combinación anterior, aunque sigue habiendo una merma considerable en ambas propiedades. El valor más alto en ductilidad y resistencia lo presentó la probeta 1 del depósito 7 , que se acercó bastante al límite elástico; incluso, se aprecia una cierta curvatura al final, lo que puede significar el inicio de la transición entre la región elástica y plástica del material. Sin embargo, no se percibe un comportamiento más allá del límite elástico, y sus curvas presentan una similitud con las de un material frágil. Al haber fractura por debajo de la temperatura equicohesiva, se puede considerar como un fenómeno de fragilización (Espejo Mora \& Hernández Albañil, 2017) y se relaciona 
directamente con la alteración no controlada de variables como distancia de arco y velocidad de avance, que, si bien se consideraron constantes, en realidad, posiblemente no lo fueron. Estas variaciones tienen una incidencia directa en el proceso de solidificación por la modificación de la tasa de enfriamiento y la relación $\mathrm{G} / \mathrm{R}$, haciendo que aspectos como la segregación por subenfriamiento constitucional también se vean alterados (Kou, 2003).

Tabla 8

Resumen esfuerzo último y alargamiento de la combinación con metal de aporte y electrodo punto violeta

\begin{tabular}{c|c|c|c}
\hline \multicolumn{4}{|c}{ CAVI } \\
\hline Depósito & Muestra & UTS [MPa] & Alargamiento \\
\hline 2 & T1 & 129,51 & $2,19 \%$ \\
\hline 2 & T2 & 145,50 & $2,47 \%$ \\
\hline 7 & T1 & 240,90 & $3,43 \%$ \\
\hline 7 & T2 & 166,10 & $2,58 \%$ \\
\hline
\end{tabular}

Fuente: elaboración propia.

Las superficies de fractura presentaron una textura granular a 200 aumentos, de acuerdo con las figuras $8(a)$ y $8(b)$, con visible reflectividad y rugosidad, lo que, junto con el tipo de carga aplicada de tensión, presentada en la figura 7, permite señalar que se trató de una posible fractura súbita frágil. En esta ocasión, no hubo coloración relacionada con corrosión, pero sigue habiendo una concentración de poros, aunque de menor tamaño, si se compara con la anterior combinación. En ambas superficies de fractura se presentan marcas de río, que indican que hay diferentes niveles en el frente de propagación de la grieta, aunque aún hay una superficie consistente con un modo de carga I, como el que fue aplicado.

Con un aumento de 500, como el de la figura $8(c)$, es posible ver en detalle la concentración de poros de diversos tamaños, grietas y microgrietas en los límites de solidificación y los conocidos poros de contracción, también producto de la solidificación. En esta imagen es posible apreciar el ya nombrado desnivel de la superficie de fractura, que presenta los mismos defectos de la superficie más cercana. En esta micrografía se presenta una trayectoria mayoritariamente intergranular.

En la figura 8(d), de 1.000 aumentos, se ve cómo, en efecto, la trayectoria de la fractura fue intergranular, pues se advierten ciertos huecos o vacíos en límite de grano, fenómeno conocido como cavitación en límite de grano. Respecto al tipo de fractura por agrietamiento, se ve tanto el tipo $D$, por lugares con apariencia de canasta de huevos, como el tipo D+F, por tratarse de un punto intermedio entre el dendrítico y el plano.

Con una imagen de 3.000 aumentos, como la de la figura $8(\mathrm{e})$, es posible notar cómo se trató de una descohesión en los límites de grano, y cómo hubo elementos de segunda en los vacíos de dichos límites. En esta imagen, es visible que hay zonas de la superficie de fractura cubiertas por una película de lo que puede ser un elemento fragilizante y es evidente la irregularidad en el llenado de los huecos de solidificación en el rango de temperatura frágil.

Analizando la composición química elemental de dos puntos cercanos sobre película que se presentó en la superficie de fractura, tal como se indica en la figura 8(f), se nota que ambos son casos de eutécticos, con un porcentaje mayor de cobre para el punto 1 , de acuerdo con la tabla 9, y con la presencia de hierro y manganeso. Cabe resaltar que, en este aspecto, el contenido de magnesio se vio mermado, hecho atribuible a que el contenido de silicio fue mayor al $1 \%-a$ causa del metal de aporte-y la relación $\mathrm{Mg} / \mathrm{Si}$ se hizo menor, alrededor de $0,5 \%$, por lo que hubo más silicio para producir la reacción y formación del compuesto $\mathrm{Mg}_{2} \mathrm{Si}$, en el cual el remanente reaccionó con hierro y manganeso, toda vez que el hierro es una impureza común en las aleaciones de aluminio.

Sin embargo, el alto contenido de hierro lo convierte en un elemento que forma compuestos fragilizantes y que, a su vez, reduce el contenido de cobre soluble para formar otros compuestos, por lo que puede tratarse de uno de los motivos por el cual la resistencia se vio drásticamente reducida -tabla 10-. Los respectivos espectros de composición elemental por EDS de los puntos 1 y 2 se encuentran en las figuras 9 y 10 , en las cuales se evidencia que todos los elementos químicos fueron considerados en el análisis. 


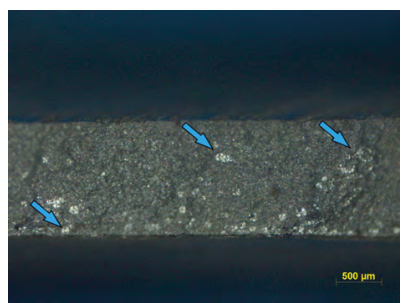

(a)

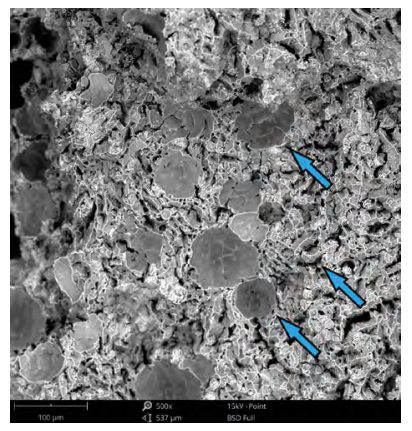

(c)

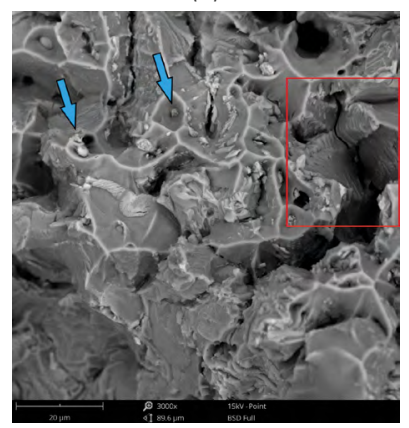

(e)

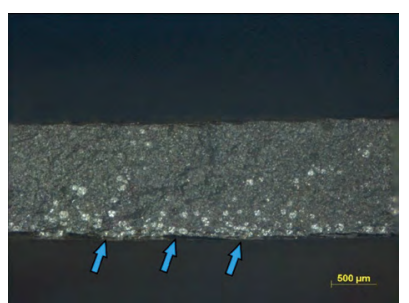

(b)

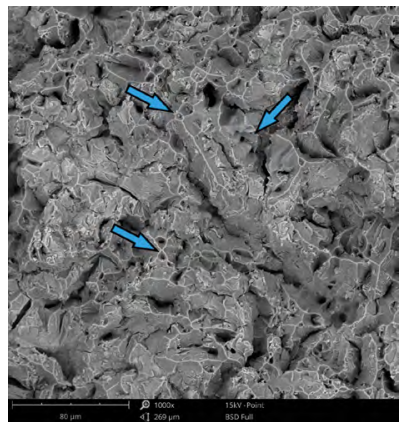

(d)

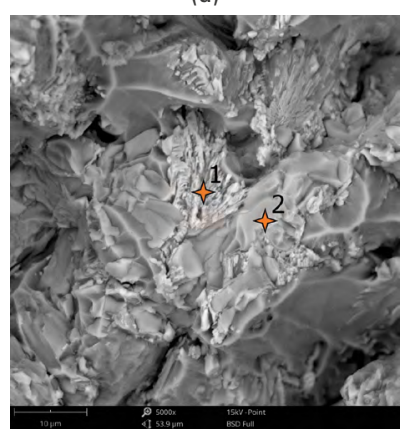

(f)
Figura 8. Superficies de fractura de la combinación con metal de aporte y electrodo punto violeta

Nota. Descripción de las imágenes: (a) macrografía de la superficie de fractura de la probeta 2 a 200X; (b) macrografía de la superficie de fractura de la probeta 7 a 200X; (c) micrografía de la superficie de fractura de la probeta 2 a 500X; (d) micrografía de la superficie de fractura de la probeta 2 a 1.000X; (e) micrografía de la superficie de fractura de la probeta 2 a 3.000X; (f) micrografía de la superficie de fractura de la probeta 7 a 5.000X, con la indicación de los puntos para análisis mediante SEM/EDS.

Fuente: elaboración propia.

Tabla 9

Composición química elemental del punto 1 de la superficie de fractura de la combinación con metal de aporte y electrodo punto violeta

\begin{tabular}{l|c|c|c}
\multicolumn{2}{c|}{ Elemento Químico } & \multicolumn{2}{c}{ Concentración } \\
\hline Nombre & Símbolo & Atómica \% & Peso \% \\
\hline Aluminio & $\mathrm{Al}$ & 76,08 & 60,26 \\
\hline Cobre & $\mathrm{Cu}$ & 19,59 & 36,53 \\
\hline Magnesio & $\mathrm{Mg}$ & 3,23 & 2,31 \\
\hline Silicio & $\mathrm{Si}$ & 1,10 & 0,91
\end{tabular}

Fuente: elaboración propia.

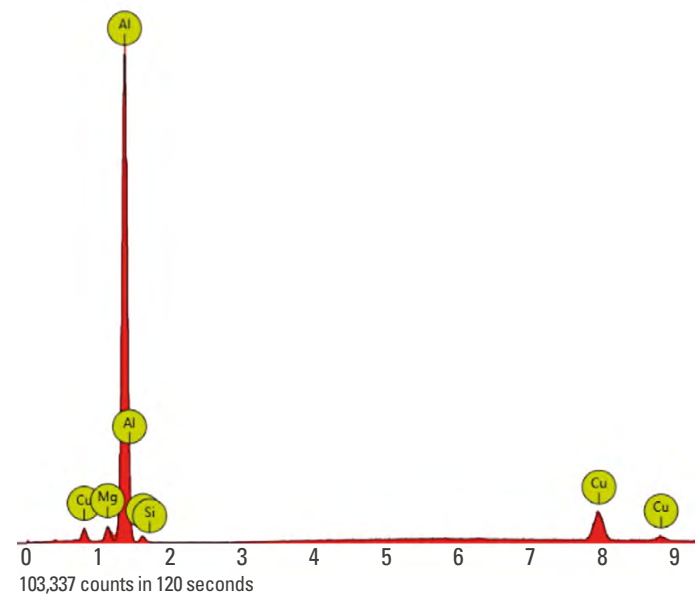

Figura 9. Espectro SEM/EDS del punto 1 de la superficie de fractura de la combinación con metal de aporte y electrodo punto violeta

Fuente: elaboración propia.

Tabla 10

Composición química elemental del punto 2 de la superficie de fractura de la combinación con metal de aporte y electrodo punto violeta

\begin{tabular}{l|c|c|c}
\multicolumn{2}{c|}{ Elemento Químico } & \multicolumn{2}{c}{ Concentración } \\
\hline Nombre & Símbolo & Atómica \% & Peso \% \\
\hline Aluminio & $\mathrm{Al}$ & 78,81 & 64,47 \\
\hline Cobre & $\mathrm{Cu}$ & 6,28 & 12,11 \\
\hline Hierro & $\mathrm{Fe}$ & 7,09 & 12,01 \\
\hline Manganeso & $\mathrm{Mn}$ & 6,08 & 10,12 \\
\hline Magnesio & $\mathrm{Mg}$ & 1,74 & 1,28 \\
\hline
\end{tabular}

Fuente: elaboración propia.

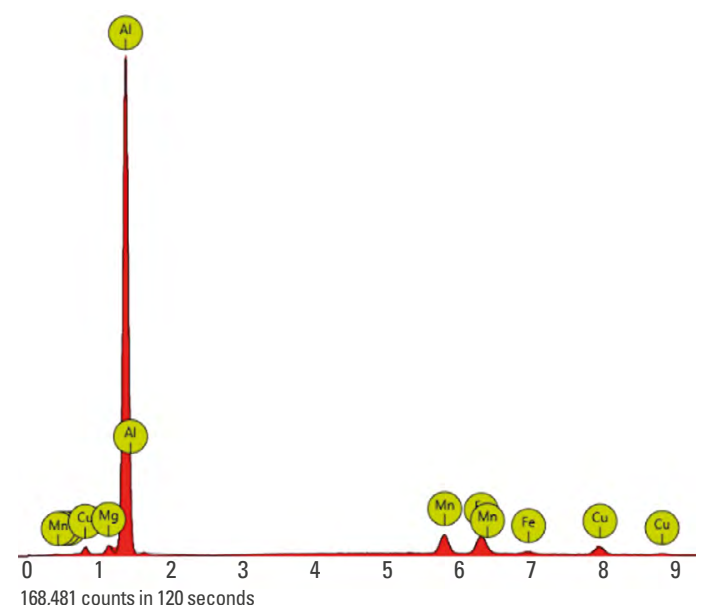

Figura 10. Espectro SEM/EDS del punto 2 de la superficie de fractura de la combinación con metal de aporte y electrodo punto violeta Fuente: elaboración propia. 


\section{Depósitos con electrodo punto violeta y sin metal aporte (SAVI)}

El resultado de los ensayos de tracción para las probetas 3 y 5 , correspondientes a la combinación de electrodo no consumible punto violeta sin metal de aporte, se encuentra en las curvas de esfuerzo-deformación de la figura 11 y en la tabla 11.

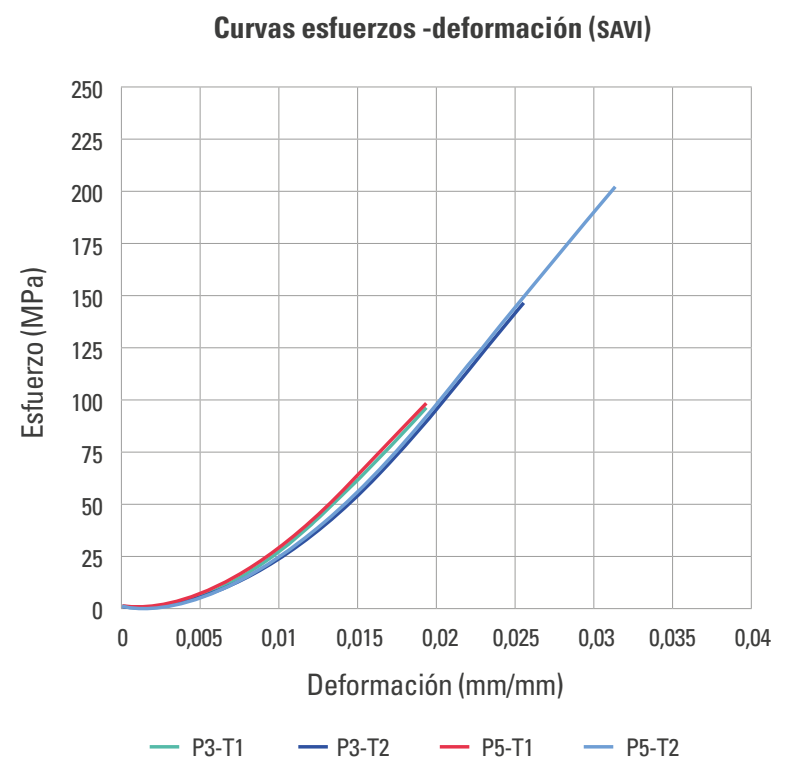

Figura 11. Curvas esfuerzo-deformación de la combinación sin metal de aporte y electrodo punto violeta

Fuente: elaboración propia.

Respecto a la combinación anterior, se presenta un comportamiento similar, en el cual las curvas siguieron el mismo recorrido en su parte elástica, pero con evidentes variaciones en resistencia mecánica y ductilidad. En general, los valores fueron mucho menores, a excepción de la probeta 2 del depósito 5 , que presentó un esfuerzo máximo de $200 \mathrm{MPa}$, con una elasticidad aproximada de 3,17\%. De nuevo, estas diferencias en la resistencia última entre las probetas se atribuyen principalmente a posibles variaciones no programadas de parámetros en la ejecución del procedimiento de soldadura, como las mencionadas en la sección anterior, afectando así el proceso de solidificación y modificando las propiedades finales (Liu \& Kou, 2017). Al igual que en los casos anteriores, no hay un límite elástico definido y las curvas se asemejan a las de materiales frágiles, lo cual, complementado con la idea anterior, muestra que la solidificación fuera de equilibrio hará cambiar el rango de temperatura frágil, que se refleja en el comportamiento mecánico del depósito (Lippold, 2015; Lippold, Böllinghaus, \& Cross, 2011).

Tabla 11

Resumen esfuerzo último y alargamiento de la combinación sin metal de aporte y electrodo punto violeta

\begin{tabular}{c|c|c|c}
\hline \multicolumn{4}{c}{ SAVI } \\
\hline Depósito & Muestra & UTs [MPa] & Alargamiento \\
\hline 3 & T1 & 94,61 & $1,90 \%$ \\
\hline 3 & T2 & 142,37 & $2,55 \%$ \\
\hline 5 & T1 & 97,79 & $1,91 \%$ \\
\hline 5 & T2 & 200,87 & $3,17 \%$ \\
\hline
\end{tabular}

Fuente: elaboración propia.

Al comparar las superficies de fractura de las probetas con mejor y peor desempeño -figuras 12(a) y 12 (b), respectivamente-, es claro por qué hubo diferencia: mientras que en la probeta 1 del depósito 3 se ve una alta concentración de porosidad de diferentes tamaños, distribuida de forma uniforme en el cordón, en la probeta 2 del depósito 5 , la porosidad es visiblemente menor en tamaño, pero con alta concentración. Ese menor tamaño con alta concentración de porosidad es el causante de que, si bien hubo un alto valor de resistencia, este estuvo muy por debajo del valor nominal.

Las superficies de fractura presentan una textura granular con apreciable brillo y rugosidad; no se observa deformación ni distorsión en la superficie, pero sí coloración relacionada con corrosión, como se muestra en la figura 12(a). En la figura 12(b) se ve una marca radial, indicación de que hubo cambio de nivel de la superficie. En general, sigue una superficie de fractura del tipo súbita frágil bajo un modo de carga tipo I. 
Con una observación a 500 aumentos, como en la figura 12(c), es visible la concentración de porosidad de gas en la superficie de fractura, junto con porosidad de contracción, que se complementan con un alto número de grietas en límite de grano. En su mayoría, se presenta una trayectoria intergranular con aspecto rocoso, que se asocia con una fractura súbita frágil. De nuevo, la superficie muestra una película no uniforme de eutéctico, que puede ser el causante de la reducción en las propiedades mecánicas.

De la figura 12(d) a 1.000 aumentos y de la figura 12 (e) a 3.000 aumentos, se puede concluir que se formó un tipo de fractura tipo $F$ y tipo $D$, de acuerdo con la clasificación de Matsuda (Lippold, 2015), con las visibles grietas que estas acarrearon. También, se corrobora el supuesto de una trayectoria intergranular, con una evidente descohesión en los límites de grano y cavitación en estos, con presencia de partículas de segunda fase.

Al analizar dos películas de eutéctico sobre la superficie de fractura que, por su tonalidad, parecen ser diferentes entre sí, tal como se aprecia en los puntos 1 y 2 de la figura 12 (f), dichas películas se asocian a compuestos de segunda fase del tipo Al-Cu-Mg, siendo diferentes, como se presumía, por la diferencia en la relación $\mathrm{Cu} / \mathrm{Mg}$ (0,62 para el punto 1 y 2,42 para el punto 2), que es esperable, dada la cantidad de elementos que se hallaron en cada caso, reduciendo el contenido de cobre soluble, tal como se observa en las tablas 12 y 13 y los respectivos espectros de composición de las figuras 13 y 14.

Sobre la misma superficie de fractura, se analizó un punto 3 , cuya composición se presenta en la tabla 14 , y el espectro de composición elemental se muestra en la figura 15. Dado el porcentaje de composición de hierro y cromo, se asocia esta partícula como una impureza de baja solubilidad, con alta incidencia en la fragilización, especialmente por su tamaño. Cabe recordar que una forma de limitar la formación de este tipo de elementos fragilizantes radica en modificar el contenido de silicio, de tal forma que este retenga el contenido de hierro (Hatch, 1984).

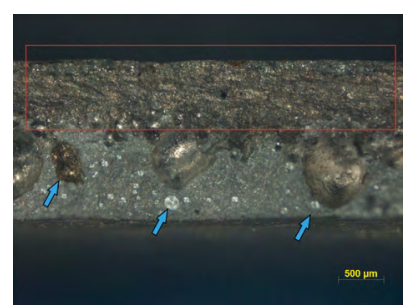

(a)

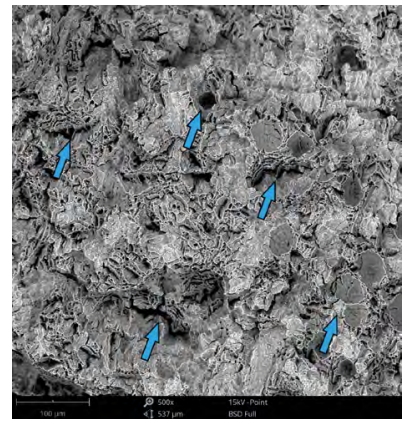

(c)

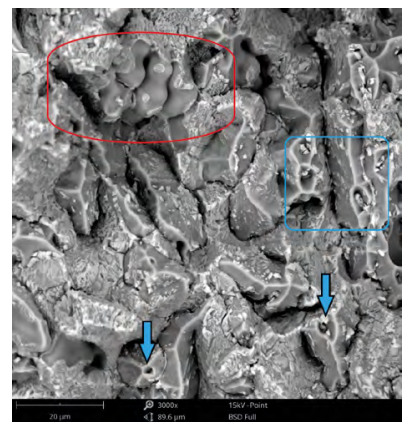

(e)

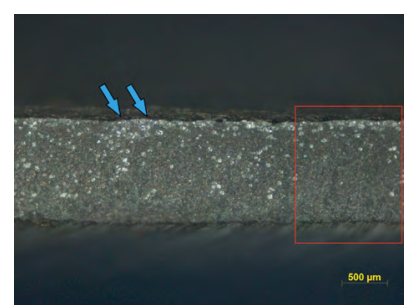

(b)

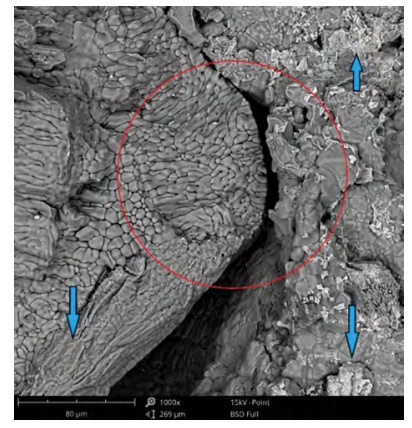

(d)

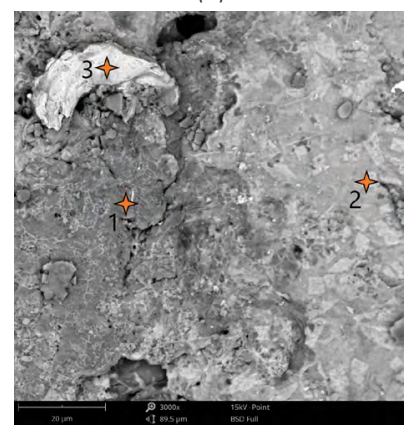

(f)

Figura 12. Superficies de fractura de la combinación sin metal de aporte y electrodo punto violeta

Nota. Descripción de las imágenes: (a) macrografía de la superficie de fractura de la probeta 3 a 200X; (b) macrografía de la superficie de fractura de la probeta 5 a 200X; (c) micrografía de la superficie de fractura de la probeta 5 a 500X; (d) micrografía de la superficie de fractura de la probeta 3 a 1.000X; (e) micrografía de la superficie de fractura probeta 5 a $3.000 X$; (f) micrografía de la superficie de fractura probeta 3 a 5.000X, con la indicación de los puntos para análisis mediante SEM/EDS.

Fuente: elaboración propia.

\section{Tabla 12}

Composición química elemental del punto 1 de la superficie de fractura de la combinación sin metal de aporte y electrodo punto violeta

\begin{tabular}{l|c|c|c}
\hline \multicolumn{2}{|c|}{ Elemento Químico } & \multicolumn{2}{c}{ Concentración } \\
\hline Nombre & Símbolo & Atómica \% & Peso \% \\
\hline Aluminio & $\mathrm{Al}$ & 92,63 & 89,18 \\
\hline Cobre & $\mathrm{Cu}$ & 2,58 & 5,85 \\
\hline Magnesio & $\mathrm{Mg}$ & 4,21 & 3,65 \\
\hline Plata & $\mathrm{Ag}$ & 0,26 & 1,00 \\
\hline Silicio & $\mathrm{Si}$ & 0,32 & 0,32 \\
\hline Fuente: elaboración propia. &
\end{tabular}




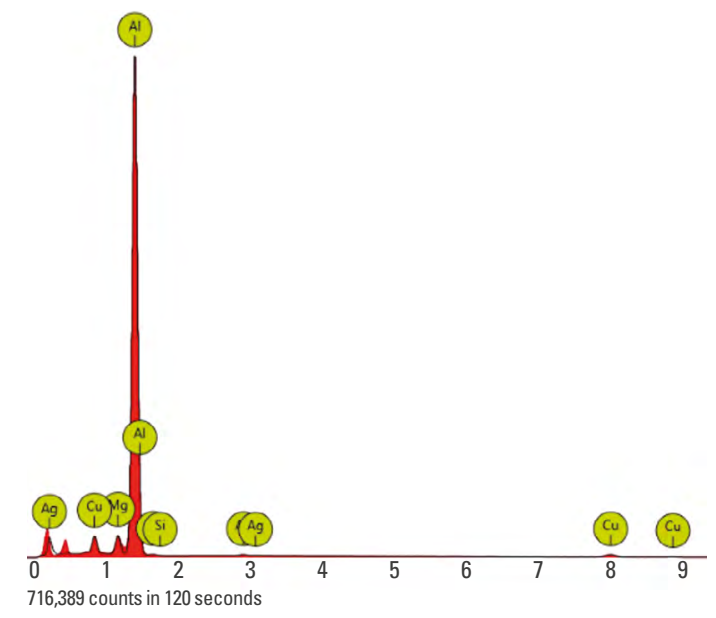

Figura 13. Espectro SEM/EDS del punto 1 de la superficie de fractura de la combinación sin metal de aporte y electrodo punto violeta

Fuente: elaboración propia.

Tabla 13

Composición química elemental del punto 2 de la superficie de fractura de la combinación sin metal de aporte y electrodo punto violeta

\begin{tabular}{l|c|c|c}
\multicolumn{2}{c|}{ Elemento Químico } & \multicolumn{2}{c}{ Concentración } \\
\hline Nombre & Símbolo & Atómica \% & Peso \% \\
\hline Aluminio & $\mathrm{Al}$ & 70,33 & 55,12 \\
\hline Cobre & $\mathrm{Cu}$ & 20,99 & 38,75 \\
\hline Magnesio & $\mathrm{Mg}$ & 8,67 & 6,12 \\
\hline
\end{tabular}

Fuente: elaboración propia.

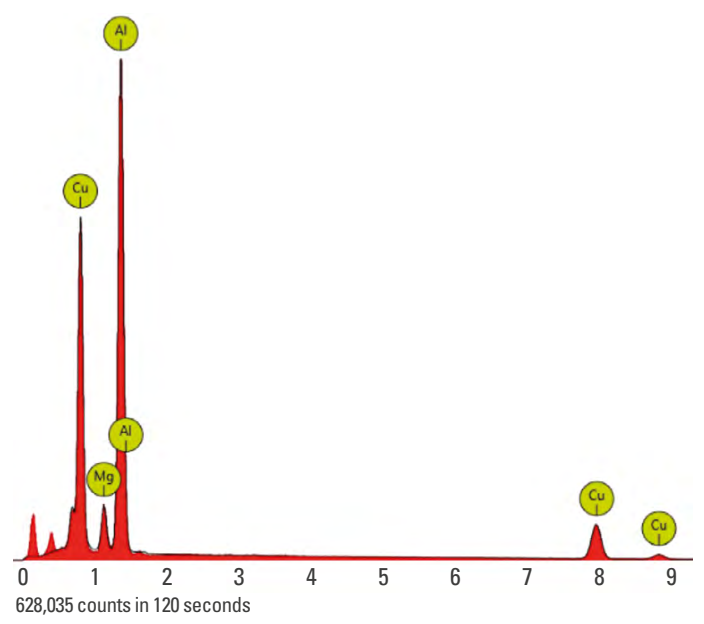

Figura 14. Espectro SEM/EDS del punto 2 de la superficie de fractura de la combinación sin metal de aporte y electrodo punto violeta Fuente: elaboración propia.
Tabla 14

Composición química elemental del punto 3 de la superficie de fractura de la combinación sin metal de aporte y electrodo punto violeta

\begin{tabular}{l|c|c|c}
\hline \multicolumn{2}{c|}{ Elemento Químico } & \multicolumn{2}{c}{ Concentración } \\
\hline Nombre & Símbolo & Atómica \% & Peso \% \\
\hline Hierro & $\mathrm{Fe}$ & 57,21 & 63,48 \\
\hline Cromo & $\mathrm{Cr}$ & 11,79 & 12,18 \\
\hline Manganeso & $\mathrm{Mn}$ & 9,83 & 10,73 \\
\hline Aluminio & $\mathrm{Al}$ & 16,09 & 8,63 \\
\hline Cobre & $\mathrm{Cu}$ & 3,19 & 4,03 \\
\hline Magnesio & $\mathrm{Mg}$ & 1,28 & 0,62 \\
\hline Silicio & $\mathrm{Si}$ & 0,61 & 0,34 \\
\hline
\end{tabular}

Fuente: elaboración propia.

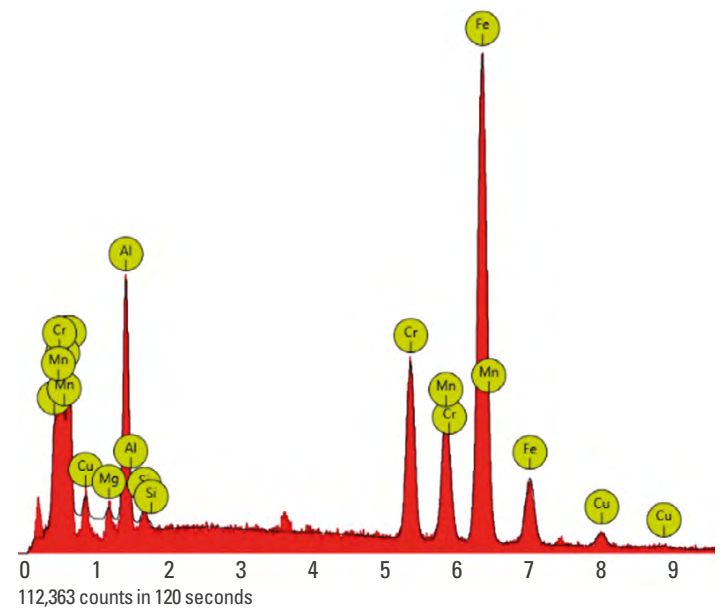

Figura 15. Espectro SEM/EDS del punto 3 de la superficie de fractura de la combinación sin metal de aporte y electrodo punto violeta Fuente: elaboración propia.

\section{Depósitos con electrodo punto verde y con metal de aporte (CAVE)}

El resultado de los ensayos de tracción para las probetas 4 y 6 , correspondientes a la combinación de electrodo no consumible punto verde con metal de aporte, se encuentra en las curvas de esfuerzo-deformación de la figura 16 y en la tabla 15.

Contrastando estos resultados con los de la combinación de electrodo punto violeta con metal de aporte (CAVI), es claro que hay una similitud en la que el valor promedio ronda los $170 \mathrm{MPa}$. Sin embargo, en este caso, no hubo un valor máximo tan marcado 
como el de $240 \mathrm{MPa}$ de la probeta 7-T1, aunque sí hubo mayor uniformidad en los valores; por ejemplo, las probetas P4-T2 y P6-T1 presentaron valores con diferencia de $5 \mathrm{MPa}$. Como fue la tendencia del ensayo, no hubo límite elástico marcado y los comportamientos fueron similares a los de un material frágil con las mismas connotaciones de los resultados anteriores.

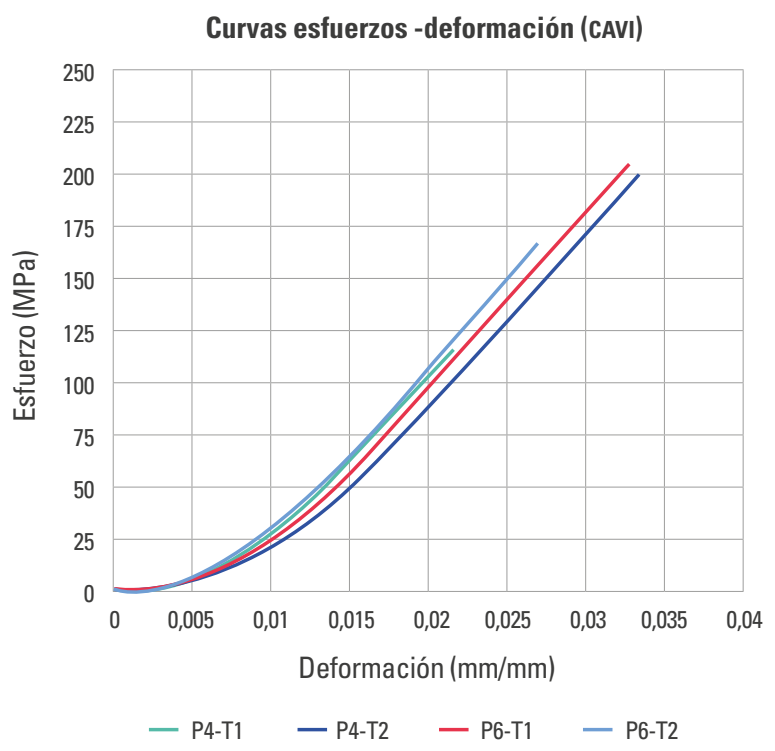

Figura 16. Curvas esfuerzo-deformación combinación con metal de aporte y electrodo punto verde

Fuente: elaboración propia.

\section{Tabla 15}

Resumen esfuerzo último y alargamiento de la combinación con metal de aporte y electrodo punto verde

\begin{tabular}{c|c|c|c}
\hline \multicolumn{4}{|c}{ CAVE } \\
\hline Depósito & Muestra & UTS [MPa] & Alargamiento \\
\hline 4 & T1 & 115,85 & $2,17 \%$ \\
\hline 4 & T2 & 199,06 & $3,33 \%$ \\
\hline 6 & T1 & 204,50 & $3,28 \%$ \\
\hline 6 & T2 & 167,10 & $2,69 \%$ \\
\hline
\end{tabular}

Fuente: elaboración propia.

Mediante una comparación de las superficies de fractura de las probetas con peor rendimiento - probeta 1 del depósito 4, figura 17(a)-y mejor rendimiento - probeta 1 del depósito 6 , figura 17 (b) - , se nota la diferencia en la concentración de porosidad, siendo nula en la segunda. En ambas se aprecia una textura granular, con una marcada reflectividad y apreciable rugosidad, rasgo distintivo de una fractura frágil de un material con baja resistencia. No se observa distorsión ni deformación, así como tampoco hay decoloraciones por corrosión. En ambas superficies hay marcas radiales a partir de la fibra media, separando dos superficies de fractura, que, de todas formas, siguen siendo del modo de carga I.

Cuando se observa la superficie de fractura de la probeta 4-T1, es clara la presencia de porosidad de gas a lo largo de esta, acompañada de grietas de solidificación y microgrietas dispersas. Se identifica un recorrido intergranular, con apariencia rocosa.

A pesar del aspecto libre de los defectos de los depósitos 6, en la figura 17 (d) se observan poros de contracción y grietas en límites de grano. También se identificó un tipo de fractura tipo $D$, de acuerdo con Matsuda (Lippold, 2015), que tiene sentido por el alto contenido de película interdendrítica que se percibe. En esta imagen, no es apreciable de forma adecuada si se trató de un modo de fractura por descohesión. Para determinarlo, basta con mirar la figura 17(e), en la que se evidencia el recorrido intergranular con presencia tanto de una transición suave como de cavitación en límite de grano, aunque en menor intensidad que en casos anteriores. De nuevo, se observa que la película cubre gran parte de la superficie de fractura, y en los agujeros de los límites de grano se aprecian partículas de segunda fase.

Tal como se hizo en las combinaciones anteriores, se evaluaron puntos específicos sobre la superficie. De acuerdo con la figura 17 (f), el primero era una película fina de eutéctico sobre el límite de grano, conclusión a la que se llegó por el alto contenido de cobre, como se presenta en la tabla 16 y su correspondiente espectro de la figura 18. Del punto 2 sobre la misma figura, por su aspecto, se pensaría que se trata de un compuesto tipo Al-Cu-Mg, pero, de acuerdo con la composición química elemental de la tabla 17 , se ve que es, en su mayoría, aluminio con bajo contenido en silicio y cobre, que se le atribuye al alto contenido de silicio que se genera por el uso de un aporte ER4043, creando un exceso de este para reaccionar con el magnesio, cobre, hierro y magnesio. El espectro de composición de este punto 2 se encuentra en la figura 19. 


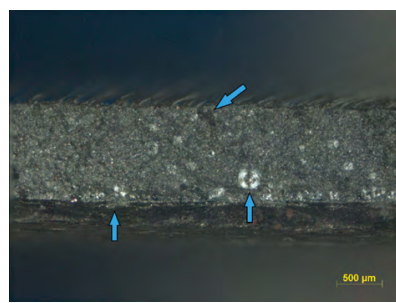

(a)

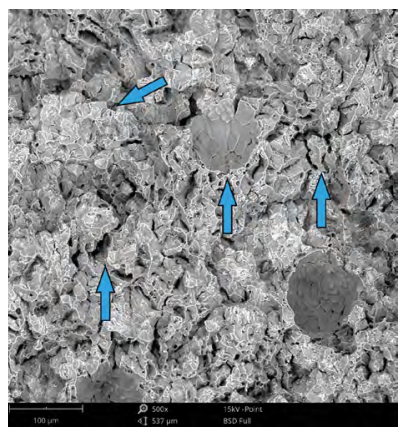

(c)

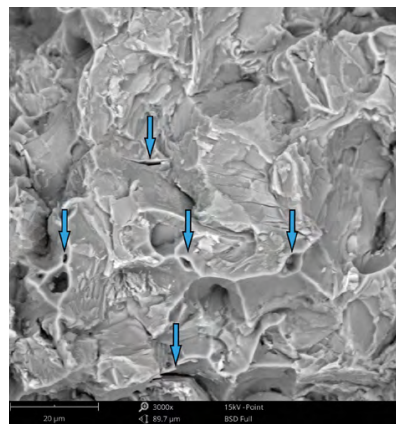

(e)

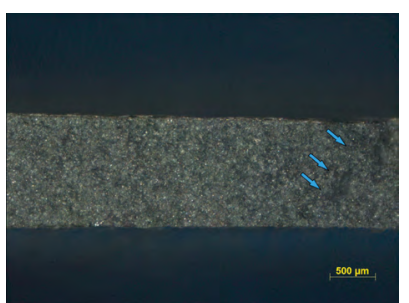

(b)

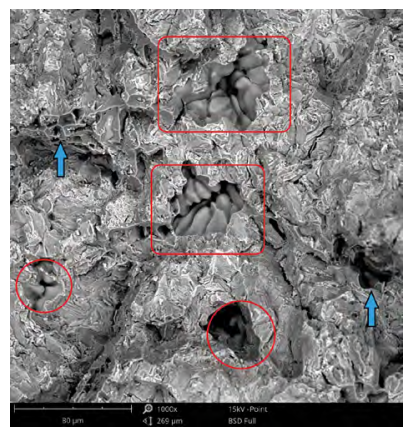

(d)

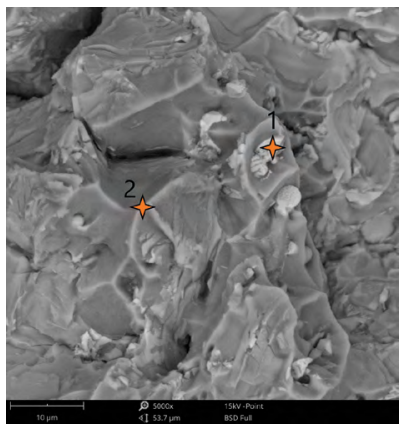

(f)
Figura 17. Superficies de fractura de la combinación con metal de aporte y electrodo punto verde

Nota. Descripción de las imágenes: (a) macrografía de la superficie de fractura de la probeta 4 a 200X; (b) macrografía de la superficie de fractura de la probeta 6 a 200X; (c) micrografía de la superficie de fractura de la probeta 4 a 500X; (d) micrografía de la superficie de fractura de la probeta 6 a 1.000X; (e) micrografía de la superficie de fractura de la probeta 4 a 3.000X; (f) micrografía de la superficie de fractura de la probeta 6 a 5.000X, con la indicación de los puntos para análisis mediante SEM/EDS.

Fuente: elaboración propia.

Tabla 16

Composición química elemental del punto 1 de fractura de la combinación con metal de aporte y electrodo punto verde

\begin{tabular}{l|c|c|c}
\multicolumn{2}{c|}{ Elemento Ouímico } & \multicolumn{2}{c}{ Concentración } \\
\hline Nombre & Símbolo & Atómica \% & Peso \% \\
\hline Aluminio & $\mathrm{Al}$ & 78,71 & 66,39 \\
\hline Cobre & $\mathrm{Cu}$ & 13,86 & 27,54 \\
\hline Silicio & $\mathrm{Si}$ & 3,60 & 3,16 \\
\hline Magnesio & $\mathrm{Mg}$ & 3,83 & 2,91 \\
\hline
\end{tabular}

Fuente: elaboración propia.

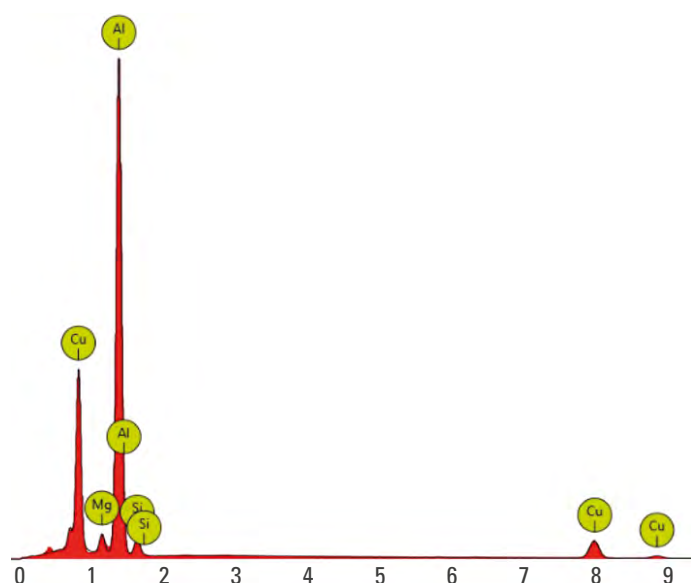

691,312 counts in 120 seconds

Figura 18. Espectro SEM/EDS del punto 1 de fractura de la combinación con metal de aporte y electrodo punto verde

Fuente: elaboración propia.

Tabla 17

Composición química elemental del punto 2 de fractura de la combinación con metal de aporte y electrodo punto verde

\begin{tabular}{l|c|c|c}
\hline \multicolumn{2}{c|}{ Elemento Químico } & \multicolumn{2}{c}{ Concentración } \\
\hline Nombre & Símbolo & Atómica \% & Peso \% \\
\hline Aluminio & $\mathrm{Al}$ & 94,58 & 93,54 \\
\hline Silicio & $\mathrm{Si}$ & 2,60 & 2,68 \\
\hline Cobre & $\mathrm{Cu}$ & 0,88 & 2,06 \\
\hline Magnesio & $\mathrm{Mg}$ & 1,93 & 1,72 \\
\hline
\end{tabular}

Fuente: elaboración propia.

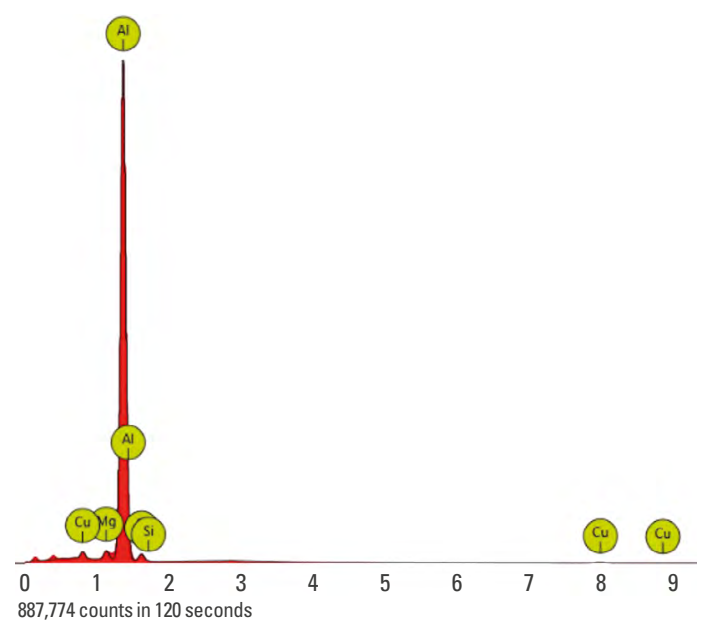

Figura 19. Espectro SEM/EDS del punto 2 de fractura de la combinación con metal de aporte y electrodo punto verde

Fuente: elaboración propia. 


\section{Discusión}

A partir de la experimentación realizada, se evidenció la existencia de un recubrimiento no uniforme sobre la superficie de fractura. Dicho recubrimiento fue, en el proceso de solidificación, un líquido eutéctico que se fracturó (Böllinghaus \& Herold, 2005). Esto está en sincronía con la afirmación según la cual, cuando un líquido se somete a una tensión hidrostática, se vuelve metaestable y, con una presión negativa lo suficientemente grande, se formarán poros finos (microporos), a causa de la cavitación de Fischer.

Asimismo, a partir de las evaluaciones, se evidenció la nula presencia del denominado curado eutéctico, lo que hace pensar que el proceso de solidificación presentó cambios en la tasa de solidificación, ligados directamente con variables como la velocidad de avance y la entrada de calor del proceso de soldadura como tal, que imposibilitaron el llenado de los intersticios de la estructura dendrítica coherente, una vez se alcanzó la temperatura de coherencia. Es por ello que, de acuerdo con la prueba de grietas y excesiva porosidad de las micrografías de fractura de las figuras 4 , 8,12 y 17 , se asume una fractura del líquido eutéctico, con posible reducción del líquido disponible, o sin la suficiente fluidez para rellenar las cavidades producidas, obteniendo agrietamiento por solidificación.

Esta situación se encuentra fuertemente ligada con las propiedades mecánicas obtenidas en las diferentes combinaciones. De acuerdo con la figura 20, y como ya se expuso en varios apartados de la sección de resultados, una solidificación por fuera de equilibrio, como es habitual en un proceso de soldadura, crea un nuevo rango de temperatura frágil (de no equilibrio), el cual produce una región en la que la resistencia mecánica y la ductilidad caen a sus valores mínimos. Si el llenado o curado eutéctico no fueron efectivos en esa etapa, no es posible la recuperación de dichas propiedades, una vez se supera la zona pastosa.

Concatenando la información de la fractografía de las cuatro combinaciones, la textura presente en todas las superficies de fractura fue granular y el recorrido predominante identificado fue el intergranular, del tipo liso o con hoyuelos por cavitación (mecanismo dúctil) del límite de grano, lo que se relaciona con una fractura súbita frágil por carga cuasiestática. En el mismo sentido, dada la evidencia de agrietamiento por solidificación, una fractura intergranular es esperable. Sin embargo, una fractura por debajo de la temperatura equicohesiva se asocia necesariamente con un fenómeno de fragilización (Espejo Mora \& Hernández Albañil, 2017). Por tanto, se llega a la conclusión de que hubo fragilización.

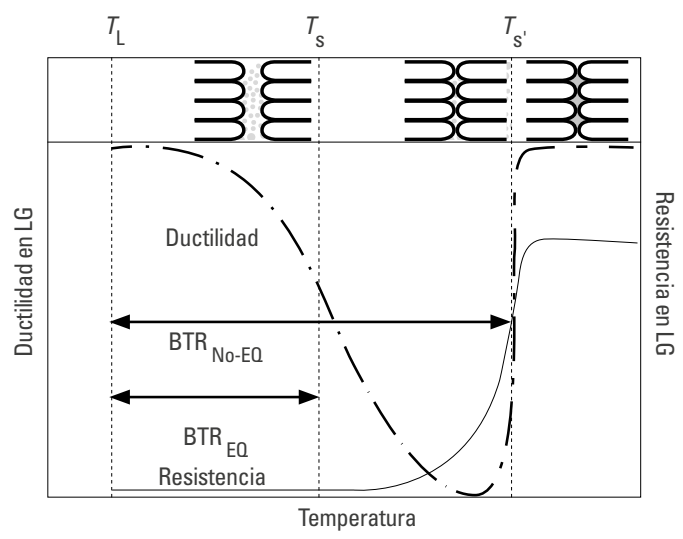

Figura 20. Curva BTR para ductilidad y resistencia en el rango de temperatura frágil de no equilibrio

Fuente: Lippold (2015, p. 130)

Adicionalmente, hechos como el que la superficie de fractura se vea granular cuando microscópicamente se tiene descohesión intergranular, o que haya una trayectoria perpendicular al modo de carga I, así como la reducción en ductilidad y resistencia en ensayos de tensión y que no haya fallo sobre la zona afectada por el calor, como es de esperarse, sino en el metal base, son indicios de fragilización por hidrógeno (Espejo Mora \& Hernández Albañil, 2017). Cabe resaltar que se considera como fragilización y no agrietamiento por hidrógeno, al tratarse de una fractura súbita con pérdida de resistencia y ductilidad ante una carga estática, o cuasiestática, como lo es un ensayo de tracción.

Los diferentes hoyuelos observados sobre las superficies de fractura hacen de la teoría de descohesión de Troiano, o de disminución de la energía superficial del metal, el mecanismo más adecuado para la investigación. Dicha teoría sostiene que el hidrógeno disuelto 
tiende a migrar hacia regiones de esfuerzo hidrostático localizado (triaxial) y debilita el material por la reducción de energía de enlace de los átomos, por lo cual la presencia del esfuerzo, en este caso a tensión, facilita la difusión del hidrógeno en el interior del elemento (ASM International, 2002; Espejo Mora \& Hernández Albañil, 2017).

Cuando la fragilización es grave a temperatura ambiente, durante un esfuerzo constante aplicado, se conoce como fragilización reversible interna por hidrógeno (ASM International, 2002). En ese sentido, una alta temperatura disuelve el hidrógeno, y, si lo llevamos al plano de la investigación, realizar un tratamiento de solución sólida, posterior a la soldadura, puede disminuir este efecto perjudicial. Al mismo tiempo, conociendo la localización de una capa de líquido eutéctico que quedó alojada sobre las superficies de fractura, junto con la identificación de compuestos a base de impurezas, como el hierro y el cromo, más una trayectoria intergranular con cavitación en límite de grano y una evidente descohesión de los granos, se llega a la conclusión de que también se presentó fractura por la fragilización dinámica.

En este tipo de situaciones, no se suele presentar deformación plástica y los límites de grano, siendo zonas de mayor difusividad, sirven como sitio de ubicación de elementos fragilizantes. Para el caso particular que aquí se trata, se considera como elemento fragilizante el mismo eutéctico o las partículas de segunda fase, a los que, debido al procedimiento de soldadura, se les otorga la difusividad necesaria para producir el fenómeno. En general, los elementos fragilizantes actuaron reduciendo la fuerza de cohesión entre los átomos del material en las zonas que se concentran, nucleándose y propagándose así el agrietamiento (Espejo Mora \& Hernández Albañil, 2017).

\section{Conclusiones}

1. Fue predominante el comportamiento bajo tensión, similar al de materiales frágiles, en el que no se presentó, en ningún momento, un límite de proporcionalidad ni un límite elástico que permitiera distinguir elasticidad y plasticidad.

2. Las superficies de fractura presentaron una textura granular, con una apreciable reflectividad y rugosidad, sin distorsiones apreciables y, en algunos casos, con decoloración, que se relacionó con corrosión. También, dichas superficies de fractura se conservaron perpendiculares al esfuerzo aplicado, que se trató como un modo de carga I, lo que se relaciona con una fractura del tipo súbita frágil.

3. La porosidad fue recurrente y varió en tamaño entre superficies: era alta en los depósitos sin metal de aporte y reducida en aquellos con metal de aporte. Esta porosidad se relaciona directamente con la solubilidad del hidrógeno en el aluminio en estado líquido.

4. Con mayores aumentos, se identificó una fractura con recorrido intergranular suave y con cavitación en límites de grano, donde predominó el modo de fractura por descohesión.

5. El análisis de composición química elemental permitió establecer que la capa que cubría la mayoría de las superficies de fractura se trató de líquido eutéctico, que presentó heterogeneidad. En el mismo sentido, no se tiene la suficiente información para determinar el mecanismo por el cual se fracturó el líquido eutéctico en el proceso de solidificación por debajo de la temperatura de coherencia, pero sí se puede establecer, por los poros y grietas formados, que este fenómeno es, en parte, el responsable de las bajas propiedades mecánicas.

6. No hubo evidencia de curado eutéctico de las grietas, posiblemente porque el líquido eutéctico no tenía la suficiente fluidez para llenar los espacios interdendríticos, o porque no fue suficiente para la estructura coherente que se tenía en el proceso de solidificación.

7. Mediante el análisis químico de los elementos que se alojaron en los hoyuelos de los límites de grano, se determinó que eran de segunda fase y que se alojaron al límite de grano por el fenómeno de nucleación y crecimiento, así como por la segregación. 
8. Con el análisis químico se determinó la presencia de compuestos formados por alto contenido de hierro y cromo, considerados como impurezas en el aluminio y que, al presentarse como compuestos, representan un riesgo en la integridad del material. Por tanto, se consideró esta situación como una potencial fragilización dinámica. Esto se sustenta en el hecho de que no se haya presentado falla por la zona afectada por el calor -esperable a causa de la recristalización-, sino por el metal de soldadura.

9. En los depósitos que tuvieron metal de aporte se evidenció un contenido de silicio mayor en el eutéctico, pero no fue el suficiente para reducir el agrietamiento en caliente.

10. La baja resistencia mecánica y la ductilidad se relacionaron con el rango de temperatura frágil de no equilibrio, que crea una zona donde ambas propiedades son mínimas.

11. Con el fin de obtener un grano refinado, que reduzca eficientemente la película de líquido intergranular, es conveniente aumentar la velocidad de avance y la entrada de calor. Este hecho puede ayudar a la reducción de la porosidad y no afectará, o será baja la afectación, del valor de subenfriamiento.

12. Por la textura, recorrido y evidente reducción en propiedades mecánicas, la fragilización se asocia, también, con un fenómeno de fragilización por hidrógeno. Esta conclusión se sustenta en la alta porosidad encontrada en todas las superficies de fractura. El mecanismo escogido fue el de la teoría de descohesión, en el cual el hidrógeno disuelto migra a regiones de esfuerzo hidrostático, debilitando el material localmente.

13. En general, los depósitos realizados con metal de aporte y con electrodo no consumible punto verde presentaron un rendimiento superior a los demás, seguidos por aquellos con metal de aporte y electrodo punto violeta.

Como trabajo futuro, se plantea migrar a un proceso automatizado, en el que variables como la velocidad de avance y la entrada de calor se mantengan constantes y no sufran fluctuaciones que ocasionen diferencias en los resultados y que, por tratarse de un proceso manual, se consideran susceptibles de no permanecer constantes en el tiempo. Otra posible modificación futura sería usar aportes con mayor contenido de silicio o de cobre, como ER4047 o ER4145, los cuales pueden ayudar a combatir la formación de grietas de solidificación, recurrentes en los análisis realizados.

Declaración de conflicto de interés: Los autores no manifiestan conflictos de interés institucionales ni personales.

\section{Referencias}

Ahn, J., Chen, L., He, E., Davies, C. M., \& Dear, J. P. (2017). Effect of filler metal feed rate and composition on microstructure and mechanical properties of fibre laser welded AA 2024-T3. Journal of Manufacturing Processes, 25, 26-36. https://doi.org/https://doi.org/10.1016/j.jmapro.2016. 10.006

Ahn, J., He, E., Chen, L., Dear, J., \& Davies, C. (2017). The effect of Ar and He shielding gas on fibre laser weld shape and microstructure in AA 2024-T3. Journal of Manufacturing Processes, 29, 62-73. https://doi.org/https://doi.org/ 10.1016/j.jmapro.2017.07.011

Ambriz, R. R., \& Jaramillo, D. (2014). Mechanical behavior of precipitation hardened aluminum alloys welds. Light Metal Alloys Applications, 35-59.

American Welding Society (2014). AWS D1.2/D1.2M: 2014 Structural Welding Code-Aluminum (6 ${ }^{\text {th }}$ Ed.). American National Standard Institute.

American Welding Society (2016). AWS B4.0:2016 - Standard Methods for Mechanical of Welds ( $8^{\text {th }}$ Ed.). American National Standards Institute.

American Welding Society (2020a). ANSI/AWS A2.4-20 - Standard Symbols for Welding, Brazing, and Nondestructive Examination. American National Standards Institute.

American Welding Society (2020b). ANSI/AWS A3.09-20 - Standard Welding Terms and Definitions. American National Standards Institute.

ASM International (2002). ASM Volume 11 - Failure Analysis and Prevention. En ASM Handbook. Materials Park.

ASM International (2020a). ASM Volume 2 - Properties and Selection: Nonferrous Alloys and Special Purpose Materials. En ASM Handbook. Materials Park. 
ASM International. (2020b). ASM Volume 6 - Welding, Brazing and Soldering. En ASM Handbook. Materials Park.

ASTM B209-14. (2014). Aluminum and Aluminum-Alloy Sheet and Plate 1. ASTM International, 1-25. https://doi.org/10. 1520/B0209-14.2

ASTM E8/E8M-21 (2021). Standard test methods for tension testing of metallic materials 1. ASTM International, 1-27. https://doi.org/10.1520/E0008

Böllinghaus, T., \& Herold, H. (2005). Hot Cracking Phenomena in Welds. Springer. https://books.google.com.co/ books?id=pLprPeLY-TOC

Cavaliere, P., Nobile, R., Panella, F. W., \& Squillace, A. (2006). Mechanical and microstructural behaviour of 20247075 aluminium alloy sheets joined by friction stir welding. International Journal of Machine Tools and Manufacture, 46(6), 588-594. https://doi.org/https://doi. org/10.1016/j.ijmachtools.2005.07.010

Chen, Y., Ding, H., Li, J., Zhao, J., Fu, M., \& Li, X. (2015). Effect of welding heat input and post-welded heat treatment on hardness of stir zone for friction stir-welded 2024-T3 aluminum alloy. Transactions of Nonferrous Metals Society of China, 25(8), 2524-2532. https://doi.org/https://doi. org/10.1016/S1003-6326(15)63871-7

Du, J., Zhao, G., \& Wei, Z. (2019). Effects of Welding Speed and Pulse Frequency on Surface Depression in Variable Polarity Gas Tungsten Arc Welding of Aluminum Alloy. Metals, 9(2), 114-134. https://doi.org/10.3390/met9020114

Esfahani, M. M., Farzadi, A., \& Zaree, S. R. A. (2018). Effect of welding speed on gas metal arc weld pool in commercially pure aluminum: theoretically and experimentally. Russian Journal of Non-Ferrous Metals, 59(1), 82-92. https://doi.org/10.3103/S1067821218010121

Espejo Mora, É., \& Hernández Albañil, H. (2017). Análisis de fallas de estructuras y elementos mecánicos. Universidad Nacional de Colombia.

Fu, R., Zhang, J., Li, Y., Kang, J., Liu, H., \& Zhang, F. (2013). Effect of welding heat input and post-welding natural aging on hardness of stir zone for friction stir-welded 2024-T3 aluminum alloy thin-sheet. Materials Science and Engineering: A, 559, 319-324. https://doi.org/https://doi. org/10.1016/j.msea.2012.08.105

Gowthaman, P. S., \& Saravanan, B. A. (2020). Determination of weldability study on mechanical properties of dissimilar Al-alloys using Friction stir welding process. $M a-$ terials Today: Proceedings. https://doi.org/https://doi. org/10.1016/j.matpr.2020.08.599
Hashimoto, T., Zhang, X., Zhou, X., Skeldon, P., Haigh, S. J., \& Thompson, G. E. (2016). Investigation of dealloying of $S$ phase (Al2CuMg) in AA 2024-T3 aluminium alloy using high resolution $2 \mathrm{D}$ and $3 \mathrm{D}$ electron imaging. Corrosion Science, 103, 157-164. https://doi.org/https://doi.org/ 10.1016/j.corsci.2015.11.013

Hatch, J. E. (1984). Aluminum Properties and Physical Metallurgy. Aluminum Science and Technology. ASM International. https://doi.org/10.1361/appm1984p001

Hima Bindu, A., Chaitanya, B. S. K., Ajay, K., \& Sudhakar, I. (2020). Investigation on feasibility of dissimilar welding of AA2124 and AA7075 aluminium alloy using tungsten inert gas welding. Materials Today: Proceedings, 26, 2.283-2.288. https://doi.org/https://doi.org/10.1016/j. matpr.2020.02.494

Jones, M. J., Heurtier, P., Desrayaud, C., Montheillet, F., Allehaux, D., \& Driver, J. H. (2005). Correlation between microstructure and microhardness in a friction stir welded 2024 aluminium alloy. Scripta Materialia, 52(8), 693-697. https://doi.org/https://doi.org/10.1016/j.scrip tamat.2004.12.027

Kou, S, \& Le, Y. (1988). Welding parameters and the grain structure of weld metal-A thermodynamic consideration. Metallurgical Transactions A, 19(4), 1.075-1.082. https://doi. org/10.1007/BF02628392

Kou, Sindo. (2003). Welding Metallurgy ( $2^{\text {nd }}$ Ed.). John Wiley \& Sons, Inc. https://doi.org/10.1016/S0016-7878(62) 80017-0

Lancaster, J. F. (1999). Metallurgy of Welding (6 $6^{\text {th }}$ Ed.). Abington Publishing.

Liang, M., Chen, L., Zhao, G., \& Guo, Y. (2020). Effects of solution treatment on the microstructure and mechanical properties of naturally aged EN AW 2024 Al alloy sheet. Journal of Alloys and Compounds, 824, 153943. https:// doi.org/https://doi.org/10.1016/j.jallcom.2020.153943

Lippold, J. C. (2015). Welding metallurgy and weldability. John Wiley \& Sons, Inc.

Lippold, J., Böllinghaus, T., \& Cross, C. E. (2011). Hot cracking phenomena in welds III. Springer Science \& Business Media.

Liu, J., \& Kou, S. (2017). Susceptibility of ternary aluminum alloys to cracking during solidification. Acta Materialia, 125, 513-523. https://doi.org/https://doi.org/10.1016/j. actamat.2016.12.028

Liu, Y., Teng, F., Cao, F. H., Yin, Z. X., Jiang, Y., Wang, S. B., \& Shen, P. K. (2019). Defective GP-zones and their evolution in an $\mathrm{Al}-\mathrm{Cu}-\mathrm{Mg}$ alloy during high-temperature aging. 
Journal of Alloys and Compounds, 774, 988-996. https:// doi.org/https://doi.org/10.1016/j.jallcom.2018.10.061

Maamar, H., Mohamed, K., Rafik, R. O., Toufik, F., Nabil, D., \& Djilali, A. (2008). Heat treatment and welding effects on mechanical properties and microstructure evolution of 2024 and 7075 aluminium alloys. Materiali in Tehnologije, 42(1), 18.

Mathers, G. (2002). The Welding of aluminium and its alloys. CRC Press.

Miller, P. L., Lyttle, K. A., Neff, J. B., Steyer, D. A., \& Pierce., K. G. (2013). Welding Gas Compositions and Method for Use (20150165565). Patent Application Publication. https://patents.justia.com/patent/20150165565\#history

Mohapatra, S., \& Sarangi, H. (2016). Comparison between tungsten inert gas and friction stir welding in commercial aluminium alloy plates. Journal of Chemical and Pharmaceutical Sciences, 2(3), 1.485-1.490.

Mondolfo, L. F. (1976). Aluminum Alloys: Structure and Properties. Butterworths.

Mouritz, A. P. (2012). Introduction to aerospace materials. Journal of the Korean Medical Association (Vol.55). Woodhead Publishing. https://doi.org/10.5124/jkma.2012.55.7.649

Norman, A. F., Drazhner, V., \& Prangnell, P. B. (1999). Effect of welding parameters on the solidification microstructure of autogenous TIG welds in an Al-Cu-Mg-Mn alloy. $M a-$ terials Science and Engineering: A, 259(1), 53-64. https:// doi.org/https://doi.org/10.1016/S0921-5093(98)00873-9

Prakash, S., Kumar, R. J. F., \& Jerome, S. (2018). Effect of heat treatment on microstructure and mechanical properties of CMT welded Aluminium alloy 2024. Materials Today: Proceedings, 5(13), 26.997-2.7003. https://doi.org/https://doi.org/10.1016/j.matpr.2018.09.003

Qi, Z., Qi, B., Cong, B., Sun, H., Zhao, G., \& Ding, J. (2019). Microstructure and mechanical properties of wire + arc additively manufactured 2024 aluminum alloy components: As-deposited and post heat-treated. Journal of Manufacturing Processes, 40, 27-36. https://doi.org/https://doi.org/10.1016/j.jmapro.2019.03.003

Soysal, T., \& Kou, S. (2019). Effect of filler metals on solidification cracking susceptibility of Al alloys 2024 and 6061. Journal of Materials Processing Technology, 266, 421-428. https://doi.org/https://doi.org/10.1016/j. jmatprotec.2018.11.022

Squillace, A., De Fenzo, A., Giorleo, G., \& Bellucci, F. (2004). A comparison between FSW and TIG welding techniques: modifications of microstructure and pitting corrosion resistance in AA 2024-T3 butt joints. Journal of Materials Processing Technology, 152(1), 97-105. https://doi.org/ https://doi.org/10.1016/j.jmatprotec.2004.03.022

Tier, M., Santos, J. F. dos, Souza Rosendo, T. de, Mazzaferro, J. A. E., Mazzaferro, C. C. P., Strohaecker, T. R., Bergmann, L., Olea, C., \& Silva, A. (2009, 13-17 de julio). A study about the mechanical properties of Alclad AA 2024 connections processed by friction spot welding 1 [conferencia]. 64. ${ }^{\circ}$ Congresso Internacional da Associação Brasileira de Metalurgia, Belo Horizonte, Brasil. https://www. researchgate.net/publication/280081104_A_Study_ About_the_Mechanical_Properties_of_Alclad_AA2024_ Connections_Processed_by_Friction_Spot_Welding_1

Vijay, S., Rajanarayanan, S., \& Ganeshan, G. N. (2020). Analysis on mechanical properties of gas tungsten arc welded dissimilar aluminium alloy (Al2024 \& Al6063). Materials Today: Proceedings, 21, 384-391. https://doi. org/10.1016/j.matpr.2019.06.136

Wang, S. B., Liu, Z. R., Xia, S. L., Key, J., \& Chen, J. H. (2017). Tetragonal-prism-like Guinier-Preston-Bagaryatsky zones in an AlCuMg alloy. Materials Characterization, 132, 139-144. https://doi.org/https://doi.org/10.1016/j. matchar.2017.08.014 